\title{
ARTíCULO
}

\section{Anatomía comparada del sistema digestivo de los lenguados Syacium papillosum y Syacium gunteri (Pleuronectiformes: Paralichthyidae)}

\author{
Comparative anatomy of the digestive system of the flounder, \\ Syacium papillosum and Syacium gunteri \\ (Pleuronectiformes: Paralichthyidae)
}

\begin{abstract}
Abraham Kobelkowsky¹ y María Iraiz Rojas-Ruiz ${ }^{2}$
${ }^{1}$ Laboratorio de Peces, Departamento de Biología, Universidad Autónoma Metropolitana, Unidad Iztapalapa, Av. San Rafael Atlixco 186, Col. Vicentina, Iztapalapa, 09349 Ciudad de México, México. akd@ xanum.uam.mx

${ }^{2}$ Licenciatura de Biología, Facultad de Ciencias, Universidad Nacional Autónoma de México, Ciudad Universitaria, Ciudad de México, México

Abstract.- In order to understand the anatomical organization of the digestive system of the flounders Syacium papillosum and Syacium gunteri, adult specimens from both sexes for the two species were analysed. By means of dissections of the visceral skeleton, visceral musculature, and the digestive tract, the morphology of the digestive system of the species was found to be affected by the extreme flattening of the body, the asymmetry of the cephalic region, and the reduction in volume of the visceral cavity. The flattening of the cephalic region implies a certain degree of vertical expansion of the visceral skeleton and musculature. The asymmetry was more pronounced in the palatine bones series, mandibles, and in the adductor mandibulae and intermandibularis muscles. The reduction in size of the visceral cavity, due to the forward extension of the anal fin, also implies that the location of the posterior intestine and anus is advanced. The most noticeable characters of the digestive tract were the great size of the stomach, the presence of 4 pyloric caeca, and the intestinal valve. The liver was located on the left side of the visceral cavity. The sharpness of the mandibular and branchial teeth, the great development of the adductor mandibulae muscles and retractor dorsalis muscles, the great size of the stomach and the intestinal ratio correspond to species of carnivorous habits. The anatomical characters that differentiate the digestive system of the species under study are the presence of a small stomach caeca, the greater length of the inferior pyloric caeca and 8 gill rakers in the inferior branch of the first branchial arch in S. papillosum, and the absence of the stomach caeca, the shorter length of the inferior pyloric caeca and 7 gill rakers in the inferior branch of the first branchial arch in S. gunteri. The comparison of the digestive system of both species with other Paralichthyidae and a Bothidae, allows the recognition of the same morphological pattern. The digestive system of Achirus lineatus (Achiridae) markedly differs from that of the Paralichthyidae and Bothidae by the extravisceral character of 2 intestinal loops, while the one from Symphurus civitatium (Cynoglossidae) differs by the presence of the suspension of the esophagus from 3 precaudal vertebrae.
\end{abstract}

Key words: Buccopharyngeal cavity, digestive tube, visceral skeleton, visceral musculature, flounders

Resumen.- Con el fin de conocer la organización anatómica del sistema digestivo de los lenguados Syacium papillosum y Syacium gunteri se analizaron ejemplares adultos de ambos sexos de las 2 especies. Mediante disecciones del esqueleto visceral, la musculatura visceral y el tubo digestivo se reconoció que la morfología del sistema digestivo de las especies está afectada por el extremo aplanamiento del cuerpo, la asimetría de la región cefálica y la reducción del volumen de la cavidad visceral. El aplanamiento de la región cefálica implica un cierto grado de ampliación en el sentido vertical del esqueleto y la musculatura viscerales. La asimetría fue más marcada en las series palatinas de huesos, las mandíbulas y en los músculos aductores mandibulares e intermandibular. La reducción del tamaño de la cavidad visceral debida a la extensión hacia adelante de la aleta anal, implica la ubicación del intestino posterior y del ano en una posición adelantada. Los caracteres más notables del tubo digestivo fueron el gran tamaño del estómago, la presencia de 4 ciegos pilóricos y de la válvula intestinal. El hígado está localizado en el lado izquierdo de la cavidad visceral. La agudeza de los dientes mandibulares y branquiales, el gran desarrollo de los músculos aductores mandibulares y retractores dorsales, el gran tamaño del estómago y la relación intestinal corresponden a especies de hábitos carnívoros. Los caracteres anatómicos que diferencian el sistema digestivo de las 2 especies en estudio, son la presencia de un pequeño ciego estomacal, la mayor longitud del ciego pilórico inferior y 8 branquiespinas en la rama inferior del primer arco branquial en S. papillosum, y la ausencia del ciego estomacal, la menor longitud del ciego pilórico inferior y 7 branquiespinas en la rama inferior del primer arco branquial en S. gunteri. La comparación del sistema digestivo de ambas especies con relación a otros paralíctidos y un bótido, permite reconocer un mismo patrón morfológico. El sistema digestivo de Achirus lineatus (Achiridae) difiere notablemente de los Paralichthyidae y Bothidae por el carácter extravisceral de 2 asas intestinales, mientras que el de Symphurus civitatium (Cynoglossidae) difiere por la presencia de la suspensión del esófago desde 3 vértebras precaudales.

Palabras clave: Cavidad bucofaríngea, tubo digestivo, esqueleto visceral, musculatura visceral, lenguados 


\section{INTRODUCCIÓN}

Los Pleuronectiformes son teléosteos comprimidos con una notable asimetría (Policansky 1982) debida a la migración ocular, manifestada en la torsión del neurocráneo, la posición de ambos ojos en el mismo lado de la cabeza (Norman 1934) y en las diferencias del sistema olfatorio entre ambos lados (Prasada Rao \& Finger 1984). En las familias Psettodidae y Citharidae los ojos pueden estar en un lado o el otro; en Scophthalmidae, Paralichthyidae, Bothidae, Achiropsettidae y Cynoglossidae los ojos se localizan en el lado izquierdo, mientras que en Pleuronectidae, Samaridae, Achiridae y Soleidae se ubican en el lado derecho (Norman 1934).

De acuerdo con Nelson (2006), Paralichthyidae es una de las familias más diversas de Pleuronectiformes, en la cual se encuentra el género Syacium Ranzani, 1842, que se distribuye en la plataforma continental de América tropical y de África occidental. Dicho género contiene 8 especies, entre las cuales 2 de ellas, Syacium papillosum (Linnaeus, 1758) y Syacium gunteri Ginsburg, 1933, se distribuyen en las costas atlánticas de Centroamérica y Sudamérica, desde Carolina del Sur hasta Río de Janeiro, y en Isla Ascensión. La distribución de $S$. gunteri es desde Florida hasta la Guyana Francesa, mientras que la de S. papillosum desde Carolina del Norte hasta Rio Grande do Sul (Figueiredo \& Menezes 2000).

Estas especies muestran un dimorfismo sexual manifestado en los machos en un mayor espacio interorbital, una mayor longitud de la aleta pectoral del lado oculado, y la presencia de 2 líneas oscuras en el margen anterior del ojo superior (Murakami \& Amaoka 1992).

De acuerdo con Nikolsky (1963), los procesos energéticos y las funciones básicas de los peces tienen lugar a expensas de la energía que entra al organismo en forma de alimento. Aunque los lenguados son peces de importancia ecológica o comercial por ser componentes de las comunidades demersales, los estudios integrales sobre su biología trófica son escasos. Entre los lenguados del Golfo de México, la anatomía general del sistema digestivo es descrita en Citharichthys spilopterus por Castillo-Rivera et al. (2000), la de Symphurus civitatium, $S$. plagiusa y S. urospilus por Kobelkowsky (2000b), la de Cyclopsetta chittendeni por Kobelkowsky (2007). La única publicación sobre alimentación de especies de Syacium es la de García-Abad et al. (1992), la cual registra la dieta de $S$. gunteri en áreas del Golfo de México.

De acuerdo con Topp \& Hoff (1972) y García-Abad et al. (1992) S. papillosum y $S$. gunteri son especies simpátricas a lo largo de la plataforma continental del Golfo de México, y cuya distribución está determinada por el tipo de sustrato, encontrándose la primera especie en sedimentos calcáreos y la segunda en sustratos lodosos y arenosos; otros factores asociados a su distribución son los bancos de camarón café (Penaeus aztecus) y camarón blanco (Peneus setiferus) (Chittenden \& McEachran, 1976). El único aspecto anatómico del sistema digestivo de Syacium corresponde a una ilustración del tubo digestivo de $S$. micrurum presentada por De Groot (1971).

Considerando que $S$. papillosum y $S$. gunteri son especies simpátricas en la plataforma continental del Golfo de México y son dominantes en las comunidades demersales, el objetivo del presente estudio fue la descripción anatómica comparativa del sistema digestivo de los lenguados S. papillosum y $S$. gunteri.

\section{MATERIALES Y MÉTODOS}

Se colectaron mediante red de arrastre ejemplares de Syacium papillosum y Syacium gunteri en localidades del litoral del Golfo de México, los cuales se fijaron con formaldehido al $10 \%$ y se conservaron en alcohol etílico al $70 \%$.

Se analizaron anatómicamente 17 ejemplares adultos de $S$. papillosum de ambos sexos, con un intervalo de longitud patrón de 100,0 a 160,5 mm, y 20 ejemplares adultos de $S$. gunteri de ambos sexos, con un intervalo de longitud patrón de 112,0 a 171,0 mm. Se observó y describió la morfología externa de la boca y de sus estructuras asociadas. Se retiró la piel de la región cefálica con el objeto de exponer la musculatura visceral, el esqueleto visceral y el neurocráneo. Se describió el arco mandibular y su dentición. Se extrajo el globo ocular inferior para evidenciar parte de los músculos viscerales más superficiales. Se retiraron el hueso nasal y el órgano del olfato para observar el conjunto de ligamentos y cartílagos rostrales.

Se analizó y retiró el músculo aductor mandibular, para exponer los músculos más internos, así como también las series de huesos del paladar y de la suspensión mandibular. Se retiraron los huesos del arco mandibular, la serie palatina, la suspensión mandibular y la serie opercular, exponiendo los aparatos hioideo y branquial y su musculatura. Se desprendieron los filamentos branquiales con el objeto de apreciar los arcos branquiales. Se observaron los músculos que relacionan la cintura escapular con los arcos branquiales y con el urohial. Posteriormente, se cortó el ligamento de Baudelot y se retiró la cintura escapular, con el propósito de exponer los músculos que relacionan las vértebras con los arcos branquiales. Se retiró el aparato branquial y se analizó su esqueleto para describir las branquiespinas y la dentición faríngea.

Se separó la piel de la región entre la cintura escapular y el nivel de las primeras espinas hemales. Se extrajeron los músculos axiales de dicha región, así como también las costillas dorsales o epipleurales y las ventrales o pleurales, con el objeto de 
descubrir la cavidad visceral. Se describió la organografía de esta cavidad. Con el fin de extraer el tubo digestivo con el hígado, la vesícula biliar, el páncreas y el bazo, se separó el esófago del último arco branquial, y se seccionaron las venas hepáticas, así como también el recto.

Se analizaron y describieron el esqueleto visceral, la dentición mandibular y branquial, la musculatura visceral, la cavidad visceral y su organografía, el tubo digestivo y el hígado. Se registró el número de branquiespinas y de ciegos pilóricos. Se determinó la relación intestinal, considerando la longitud del tubo digestivo (esófago, estómago, intestino anterior e intestino posterior) y la longitud patrón.

La terminología osteológica siguió el criterio de Hoshino \& Amaoka (1998) y la terminología miológica el criterio de Winterbottom (1974). Las ilustraciones se realizaron mediante una camera lucida acoplada a un microscopio estereoscópico Wild M3Z.

Debido a que los Paralichthyidae tienen ambos ojos en el lado izquierdo, se utilizaron los siguientes términos: lado izquierdo = lado oculado; lado derecho= lado ciego; ojo derecho $=$ ojo superior; ojo izquierdo $=$ ojo inferior.

Con el objeto de contribuir en la definición del patrón morfológico del sistema digestivo de los Paralichthyidae se realizó una comparación con otras especies de la familia como: Citharichthys spilopterus Günther, 1862, Cyclopsetta chittendeni Bean, 1895 y Paralichthys lethostigma Jordan \& Gilbert, 1884. De la misma manera, también se comparó con otras especies de Pleuronectiformes como: Bothus robinsi Topp \& Hoff, 1972 (Bothidae), Achirus lineatus (Linnaeus, 1758) (Achiridae) y Symphurus civitatium Ginsburg, 1951 (Cynoglossidae).

\section{Resultados}

\section{ESQUELETO VISCERAL}

El esqueleto visceral de Syacium papillosum y Syacium gunteri consistente en los arcos mandibular, hioideo y branquiales se localiza ventralmente al neurocráneo y muestra en conjunto un notable aplanamiento lateral y un alargamiento en el sentido vertical. Se articula con la región etmoidea del neurocráneo mediante los ligamentos rostrales y con la cápsula ótica mediante los huesos hiomandibulares y faringobranquiales 1. Su asimetría se manifiesta principalmente en las series palatinas y las mandíbulas, y en segundo término en las suspensiones mandibulares y series operculares.

Mandíbulas. La boca de $S$. papillosum y $S$. gunteri tiene orientación oblicua y no sobrepasa hacia atrás el nivel de la pupila del ojo inferior (Figs. 1a, 9a). Se reconoce una mayor longitud de las mandíbulas superior e inferior del lado ciego (Figs. 3a, b; 9a, b). Las mandíbulas superiores están formadas por los premaxilares y los maxilares, mientras que las inferiores están integradas por los dentarios, anguloarticulares, retroarticulares y coronomeckelianos (espleniales).

En las mandíbulas superiores es notable la asimetría de la sínfisis premaxilar (Fig. 3f). Asimismo, el extremo posterior del maxilar del lado oculado es más amplio y de bordes romos (Fig. 3b), mientras que el del lado ciego es más anguloso (Fig. 3a). Ambos maxilares difieren en la orientación del borde que recibe al ligamento maxilo-mandibular, siendo más lateral en el elemento del lado oculado. En las mandíbulas inferiores sobresale el carácter esbelto del proceso coronoides de ambos anguloarticulares.

Series palatinas (Figs. 2, 9c). Cada serie está integrada en ambas especies por el palatino, el endopterigoides y el ectopterigoides. Se reconocen a los palatinos como los huesos de mayor asimetría de todo el esqueleto visceral, teniendo el palatino del lado oculado el proceso articular delgado y curvo, mientras que el del lado ciego es aplanado y recto (Fig. 2c). Luego, se reconocen los endopterigoides con una marcada asimetría, consistente en la mayor inclinación del elemento del lado oculado, así como también por su concavidad que contribuye a la formación de la 'órbita ocular secundaria' que alberga al ojo inferior. El ectopterigoides es el elemento más delgado de la serie.

Suspensiones mandibulares (Figs. 2a, 9c). Cada suspensión mandibular está formada en las 2 especies por los huesos hiomandibular, metapterigoides, simpléctico y cuadrado (Fig. 2a, 9c), siendo ligeramente más larga la del lado oculado. Cada hiomandibular es alargado, tiene un cóndilo anterodorsal que se articula con el esfenótico, uno posterodorsal que lo hace con el pterótico y otro posterior que recibe al opercular; lateralmente lleva un reborde vertical que se acopla con parte del preopercular. Cada metapterigoides es irregular y se une por sutura al simpléctico, el cual es relativamente pequeño. Cada cuadrado es triangular con el cóndilo articular hacia abajo, que se acopla a la fosa articular del hueso ánguloarticular (Figs. 2a, 9c).

Series operculares (Figs. 2a, 9a, b, c). Cada serie está constituida en las 2 especies por los huesos preopercular, opercular, interopercular y subopercular y muestra una discontinuidad entre el preopercular y el resto de la serie. Se observan ligeras diferencias entre la serie del lado oculado con respecto a la del lado ciego. Cada preopercular lleva una rama del sistema sensorial de la línea lateral y está unido más firmemente a la suspensión mandibular que al resto de la serie 
opercular. Cada opercular forma un pequeño proceso anterodorsal al que se une el músculo dilatator operculi (Fig. 5a); su borde superior tiene una escotadura por la que se observa el adductor operculi, y en la parte recta del borde se inserta el levator operculi (Figs. 5a, d). El subopercular se prolonga por un delgado proceso bajo el borde inferior del opercular. El interopercular es triangular y se une mediante un ligamento al retroarticular.

Aparato hioideo (Figs. 4, 10a). Está formado en las especies en estudio por las 2 barras hioideas y por los huesos impares basihial y urohial. Cada barra hioidea es robusta y está integrada de adelante hacia atrás por los siguientes huesos (Figs. 4a, 10b): hipohial dorsal, hipohial ventral, ceratohial, epihial, interhial y los radios branquiostegos 1 a 7 . Los 3 primeros radios branquiostegos se unen por su extremo anterior al ceratohial y se curvan por la parte interna, mientras que los restantes se articulan al epihial; el último radio branquiostego se adosa a la superficie interna de los huesos interopercular y subopercular. El basihial es aplanado lateralmente y se une a cada hipohial ventral mediante ligamentos. El urohial es impar, está aplanado lateralmente y tiene forma de 'L' invertida (Figs. 4c, 10c).
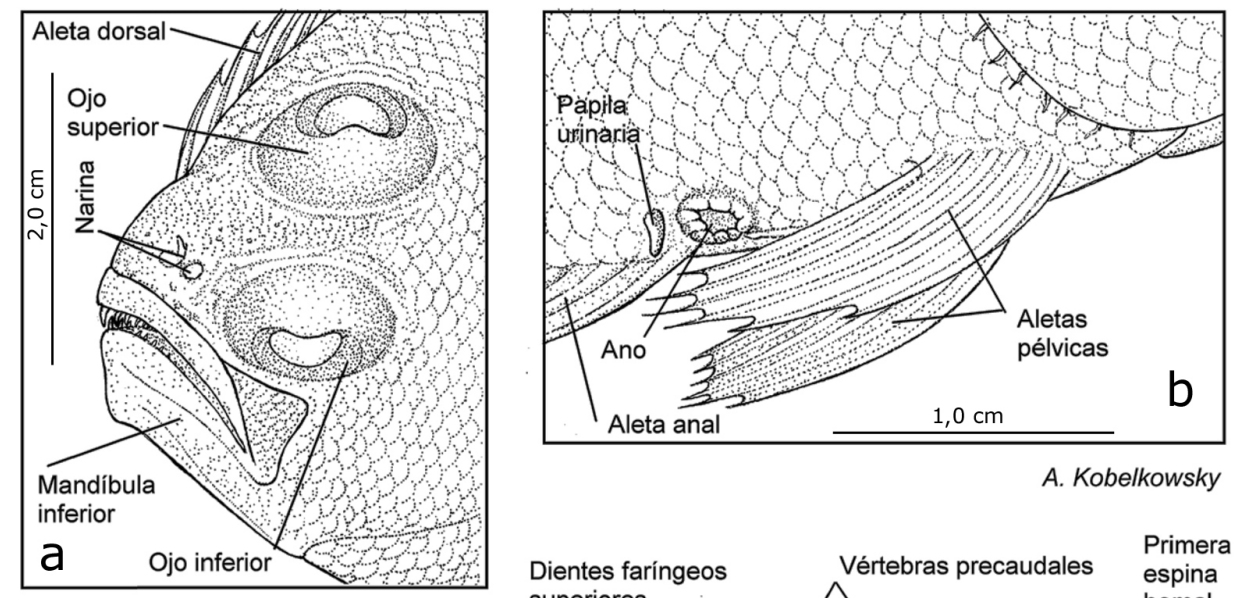

A. Kobelkowsky

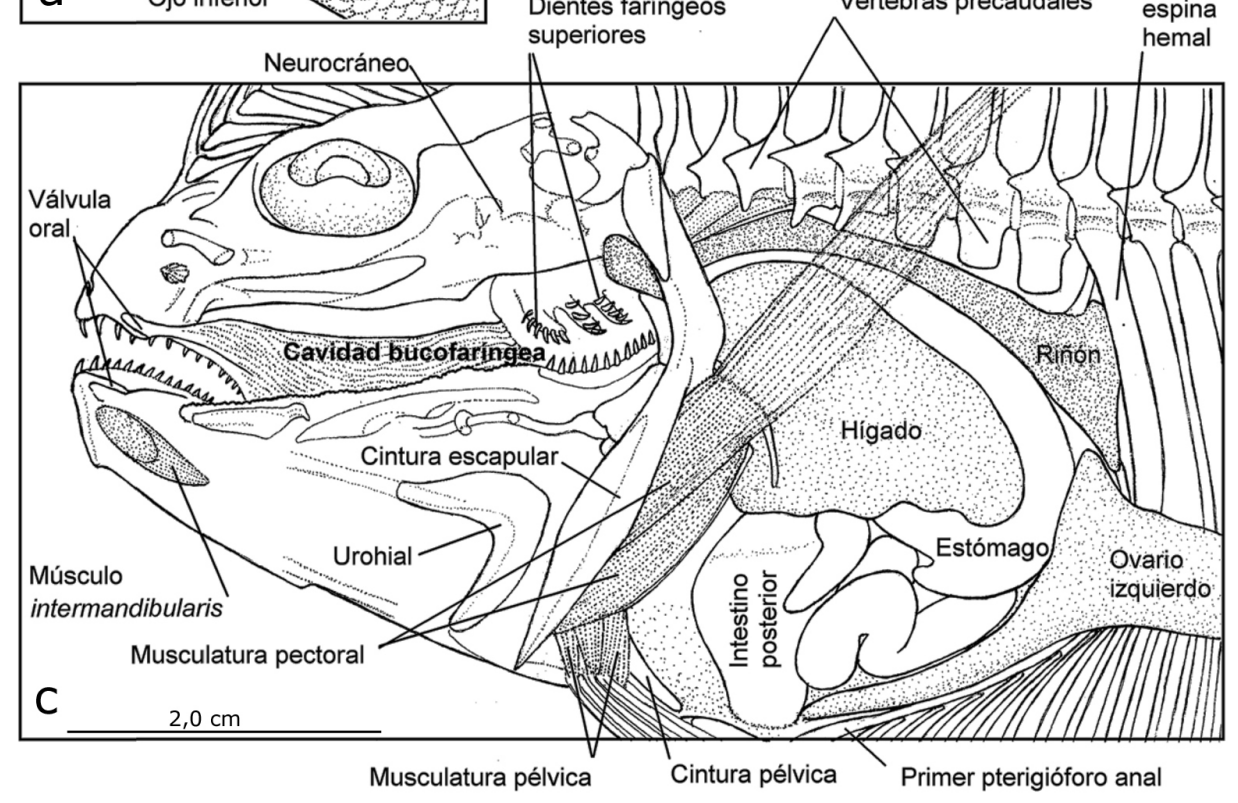

Figura 1. Sistema digestivo de un ejemplar hembra de Syacium papillosum. a) Aspecto externo de la boca por el lado oculado. b) Localización del ano y la papila urinaria en el lado ciego en la hembra, c) Vista lateral izquierda de la cavidad bucofaríngea y de la organografía de la cavidad visceral / Digestive system of Syacium papillosum. a) External view of the mouth from the eye side, b) Localization of the anus and urinary papilla on the blind side in the female, c) Left lateral view of the buccopharyngeal cavity and the organopraphy of the visceral cavity 
Aparato branquial (Figs. 4, 10a). Está formado en las 2 especies por 4 basibranquiales impares, 3 pares de hipobranquiales, 5 pares de ceratobranquiales, 4 pares de epibranquiales y 4 pares de faringobranquiales. Los hipobranquiales, ceratobranquiales y epibranquiales tienen un surco externo por el que corren las arterias branquiales aferentes y eferentes, y llevan en su borde externo las branquiespinas.
Éstas son triangulares y cada una soporta en su borde superior una hilera de dientes delgados (Figs. 4d, 10a). El primer arco branquial lleva generalmente en las 2 especies 3 branquiespinas en su rama superior (epibranquial) y 8 en su rama inferior (hipobranquial y ceratobranquial) en $S$. papillosum (Fig. 4a) y 7 en S. gunteri (Fig. 10a).
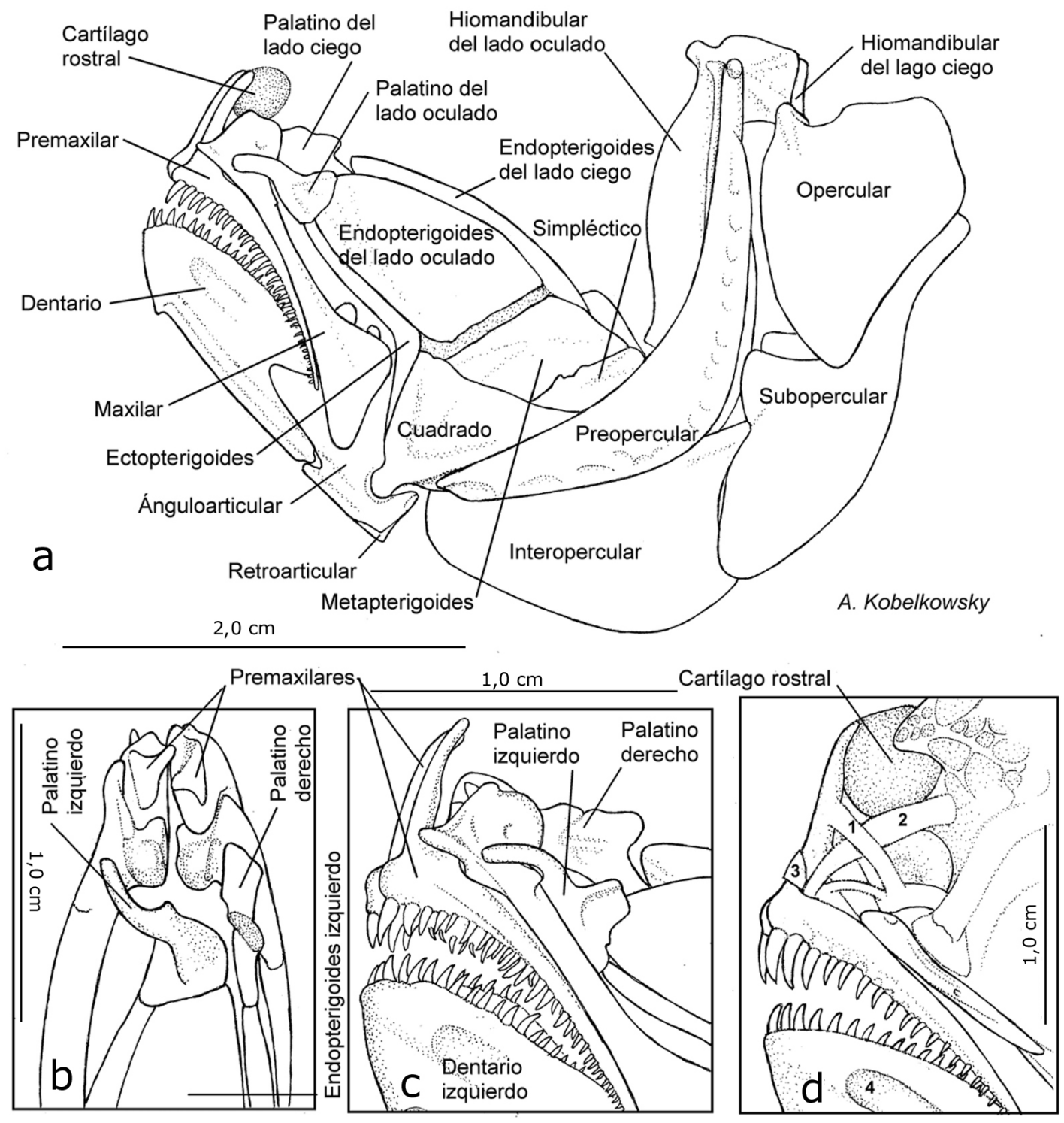

Figura 2. Esqueleto visceral de Syacium papillosum. a) Vista lateral (lado oculado) de las mandíbulas, serie palatina, suspensión mandibular y el opérculo. b) Vista dorsal de la región rostral, c) Vista del lado oculado de los huesos de la región rostral, d) Vista del lado oculado de los ligamentos rostrales: 1. Ligamento palatino-premaxilar, 2. Ligamento etmoides-premaxilar, 3. Ligamento interpremaxilar / Visceral skeleton of Syacium papillosum, a) Lateral view (eye side) of the jaws, mandibular suspension, and operculum, b) Dorsal view of the rostral region, c) Eye side view of the bones of the rostral region, d) Eye side view of the rostral ligaments: 1. Palatine-premaxillae ligament. 2. Ethmoid-premaxillae ligament. 3. Intermaxillae ligament 
De los basibranquiales, el segundo es el más largo. De los hipobranquiales el 1 muestra una escotadura en su lado interno, mientras que los restantes forman cada uno un proceso ventral que protege a la aorta ventral; el proceso ventral de cada hipobranquial 3 es largo y delgado y permite la inserción tanto del músculo transversus communis como de un ligamento que se fija en el urohial; entre el borde externo del hipobranquial 2 y cerca del borde interno del hipobranquial 1 se extiende un largo ligamento (Fig. 4b). Los ceratobranquiales 5 o 'huesos faríngeos inferiores' son angostos y soportan una hilera de dientes largos y delgados. Los faringobranquiales 1 son alargados y se articula cada uno con la cápsula ótica correspondiente (Figs. 4a, 6b), constituyendo la suspensión del aparato branquial. Los faringobranquiales 2, 3 y 4 o 'huesos faríngeos superiores' de cada lado desarrollan procesos ventrales robustos con una hilera de dientes cónicos y curvados hacia el plano sagital (Fig. 4b); la posición de los 6 procesos dentados se alterna dentro de la cavidad faríngea.

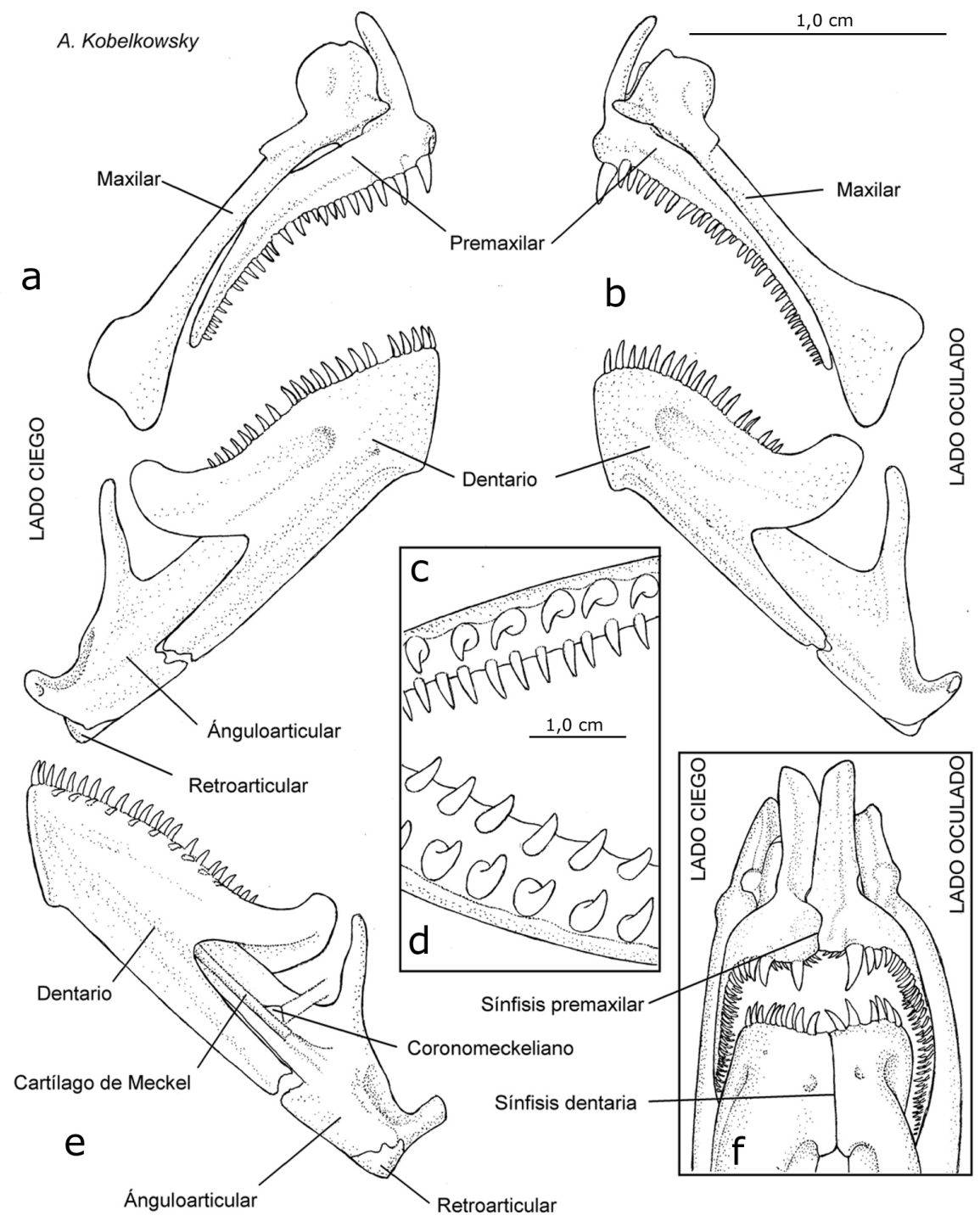

Figura 3. Mandíbulas de Syacium papillosum. a) Vista del lado ciego de las mandíbulas superior e inferior, b) Vista del lado oculado de las mandíbulas superior e inferior, c) Vista ventral de la dentición del premaxilar, d) Vista dorsal de la dentición del dentario, e) Vista interna de la mandíbula inferior del lado ciego, f) Vista anterior de las mandíbulas de ambos lados / Jaws of Syacium papillosum. a) Blind side view of the upper and lower jaws, c) Ventral view of the premaxillary dentition, d) Dorsal view of the dentary dentition, e) Medial view of the blind side lower jaw, f) Anterior view of the both side jaws 


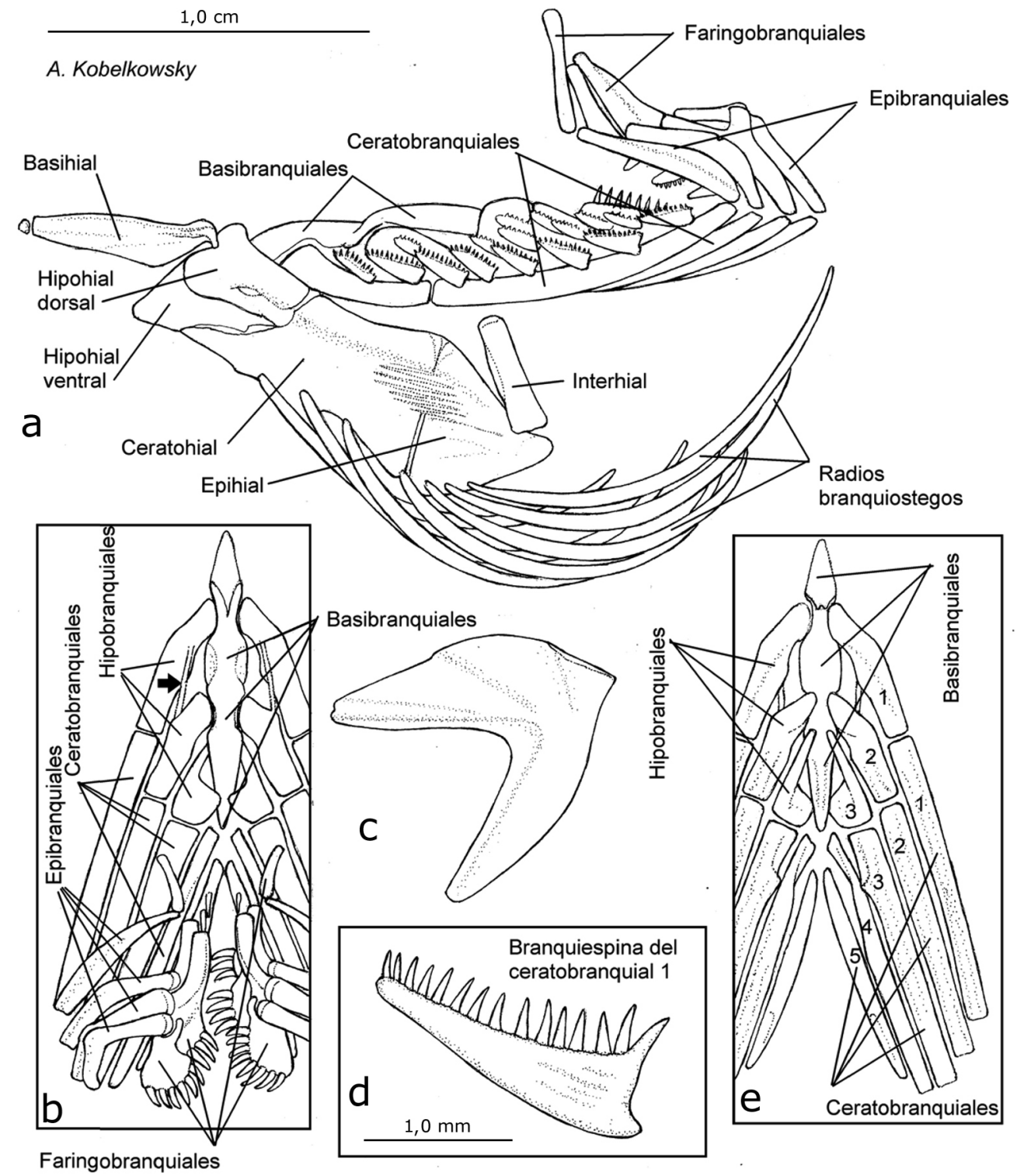

Figura 4. Aparatos hioideo y branquial de Syacium papillosum. a) Vista lateral izquierda, b) Vista dorsal del aparato branquial; la flecha señala el ligamento, c) Vista lateral izquierda del urohial, d) Vista lateral de una branquiespina del ceratohial 1, e) Vista ventral del aparato branquial / Hyoid and branchial apparatus of Syacium papillosum, a) Left lateral view, b) Dorsal view of the branchial apparatus; arrow points the ligament, c) Left lateral view of the urohyal, d) Lateral view of a gill raker of the ceratohyal 1 , e) Ventral view of the branchial apparatus

\section{DenTición}

La dentición mandibular de ambas especies de Syacium se presenta formando 2 hileras de dientes cónicos en los premaxilares (Fig. 3c) y los dentarios (Figs. 3d, 5c). Los de la hilera externa son de mediano tamaño, mientras que los de la interna son pequeños y de orientación hacia la cavidad bucal. Los dientes de la hilera interna de los dentarios son más gruesos que los correspondientes de los premaxilares.
La dentición branquial se presenta en las branquiespinas, los huesos faríngeos inferiores (ceratobranquiales 5) y los faringobranquiales $2 \mathrm{a} 4$. Cada branquiespina lleva en su borde superior una hilera de pequeños dientes (Figs. 4d, 10a). Los huesos faríngeos inferiores llevan una hilera de dientes rectos y puntiagudos (Figs. 1c, 4a). Cada uno de los faringobranquiales 2 a 4 lleva una hilera de dientes puntiagudos curvados hacia el plano sagital (Figs. 1c, 4b). 


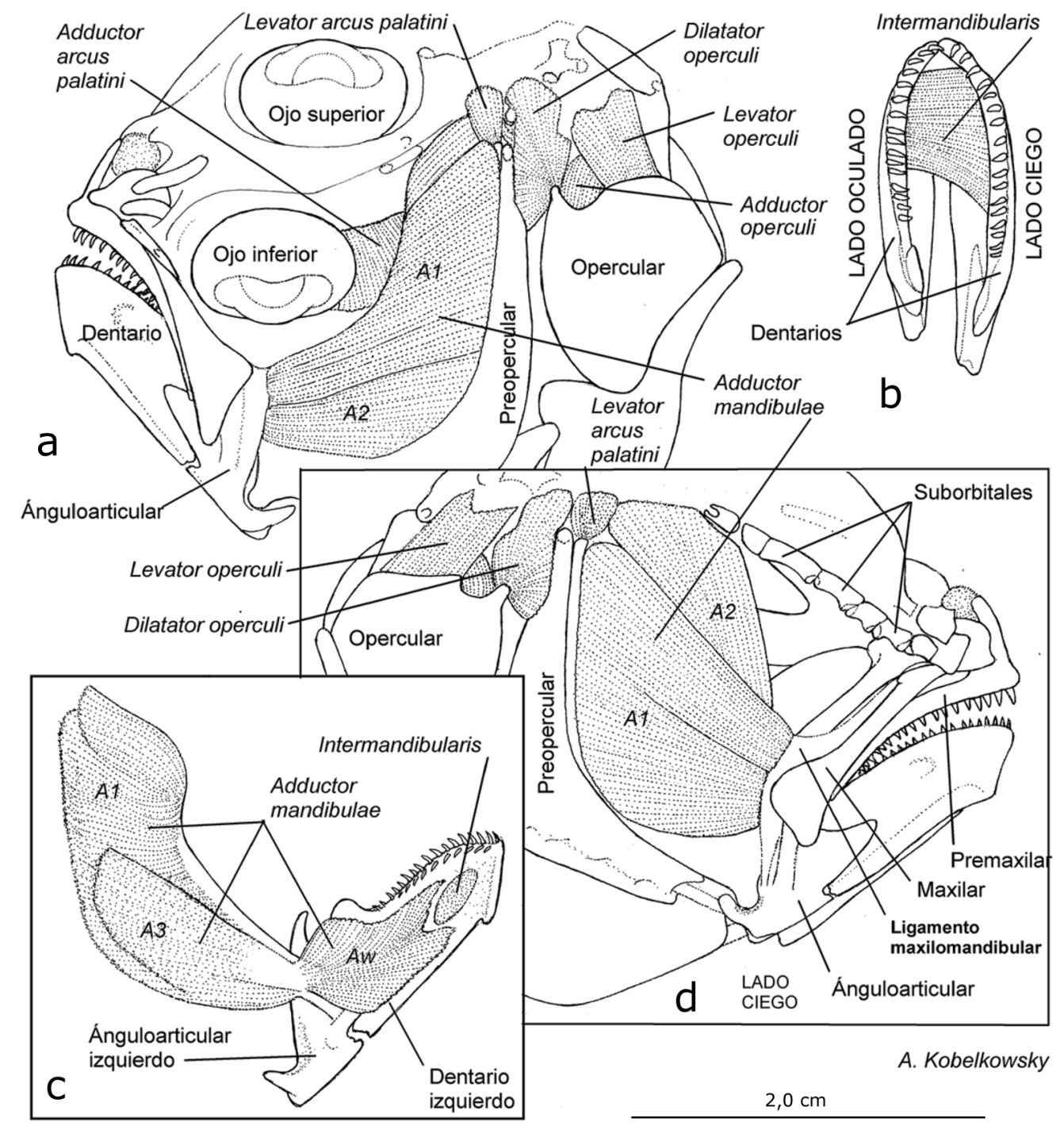

Figura 5. M usculatura visceral de Syacium papillosum. a) Vista del lado oculado del adductor mandibulae y algunos músculos operculares, b) Vista dorsal del intermandibularis, c) Vista lateral interna del adductor mandibulae del lado oculado, d) Vista del lado ciego del adductor mandibulae y músculos operculares / Visceral musculature of Syacium papillosum. a) Eye side view of the adductor mandibulae and some opercular muscles, b) Dorsal view of the intermandibularis, c) Internal lateral view of the adductor mandibulae of the eye side, d) Blind side view of the adductor mandibulae and some opercular muscles

\section{Musculatura Visceral}

La musculatura visceral de las especies de Syacium está organizada de manera general en los siguientes conjuntos de músculos: a) los que mueven las mandíbulas superior e inferior, b) los que accionan la suspensión mandibular, c) los que relacionan el opérculo con el neurocráneo, d) los que elevan las ramas superiores de los arcos branquiales, e) los relacionados con las ramas inferiores de los arcos branquiales, f) los que elevan la serie palatina, g) los que se fijan al urohial. a) El músculo adductor mandibulae (Figs. 5a, c; 9a, b) está subdividido en las secciones $A 1, A 2, A 3$ y $A w$; muestra una notable asimetría, consistente en la formación de una concavidad anterior de la sección $A l$ del músculo del lado oculado, que proporciona espacio para el ojo inferior; mientras que el homónimo del lado ciego es más amplio. El músculo intermandibularis (Figs. 5b, 6d, 10b) es un músculo que relaciona ambos dentarios, y muestra una considerable asimetría por tener el extremo del lado ciego notablemente más amplio que el del lado oculado. El protractor hyoidei (Figs. 6d, 10b) 
tiene un aspecto metamérico, se origina en el ceratohial y se inserta mediante unos tendones planos en los dentarios, cerca de su sínfisis; dicho tendón se divide en 2 ramas que rodean al intermandibularis. b) El músculo levator arcus palatini (Fig. 6a) es de apariencia compuesta; se origina del esfenótico y se inserta ampliamente en el hiomandibular.c) El dilatator operculi (Fig. 6a) tiene forma de abanico, se origina de parte del esfenótico y del preopercular y se inserta en el proceso anterodorsal del opercular. El adductor operculi (Fig. 6a) se origina del proótico y se inserta en la superficie interna del opercular inmediatamente detrás del proceso superior. El levator operculi (Fig. 6a) se origina del postemporal y se inserta en parte del borde dorsal del opercular, detrás del adductor operculi. d) Los levatores externi (Figs. 6b, 10b) se originan del proceso del esfenótico y se insertan en los epibranquiales 1 a 4. Los levatores interni 1 y 2 (Figs. 6b, c) se originan de la superficie interna del extremo superior del hiomandibular y se insertan en los faringobranquiales 2 y 3 , respectivamente, mientras que el levator interni 3 se origina del proceso lateral del esfenótico y se inserta en el faringobranquial 4. Como músculos intrínsecos del aparato branquial se identifican a los transversi dorsales, al obliquus dorsalis y a los adductores (Fig. 6c). El retractor dorsalis (Figs. 6a, b; 9a, 10b) está constituido por 4 paquetes musculares que se originan respectivamente de los centros vertebrales 3 a 6 y se insertan mediante un tendón laminar en los faringobranquiales 3 y 4. e) El pharyngoclavicularis internus (Figs. 6d, 10b) es corto y robusto, se origina en el ceratobranquial 5 y se inserta en la superficie anterior de la porción media del cleitrum. El pharyngoclavicularis externus (Figs. 6d, 10b) es largo y aplanado, se origina del ceratobranquial 5 y se inserta en la superficie anterior de la porción inferior del cleitrum. f) El músculo adductor arcus palatini (Fig. 6a) se origina del paraesfenoides y se inserta tanto en la superficie superior del endopterigoides como en parte de la superficie interna del metapterigoides y del hiomandibular. g) El sternohyoideus (Figs. 6d, 10b) es corto y notablemente robusto, tiene aspecto metamérico, se origina de manera amplia de la superficie anterior de la porción inferior del cleitrum y se inserta en ambas caras del urohial.
Figura 6. Musculatura visceral de Syacium papillosum. a) Músculos del paladar, suspensión mandibular y opérculo del lado oculado, y parte del retractor dorsalis, b) Músculos dorsales de los arcos branquiales del lado oculado, c) Vista dorsal de los músculos de los arcos branquiales, d) Vista del lado oculado de las relaciones musculares de las mandíbulas con la barra hioidea y de la cintura escapular con los arcos branquiales y con el urohial / Visceral musculature of Syacium papillosum. a) Muscles of palatine, mandibular suspension and operculum of the blind side, c) Dorsal view of the muscles branchial arches, d) Eye side view of the muscular relationships of the jaws and with the hyoid bar, and of the scapular girdle with the branchial arches and urohyal

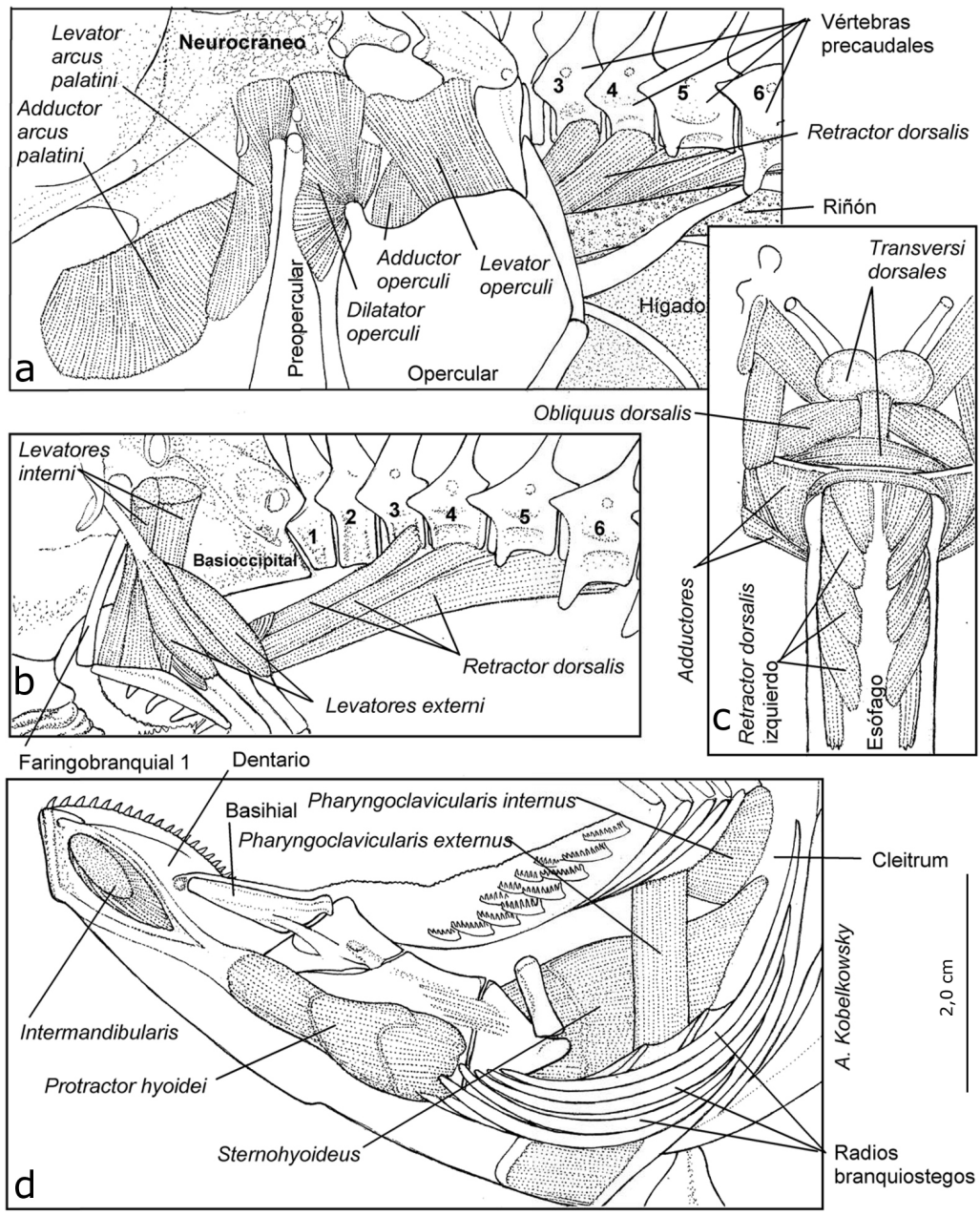

Vol. 52, N², 2017 


\section{Cavidad visceral}

La cavidad visceral de $S$. papillosum y $S$. gunteri es relativamente reducida (Figs. 1c, 9a) y está aplanada lateralmente. Está delimitada por las cinturas escapular y pélvica, las vértebras precaudales, la primera espina hemal, el primer pterigióforo de la aleta anal, las costillas pleurales, las musculaturas pectoral y pélvica y los miotomos precaudales. La espina hemal de la primera vértebra caudal es larga y robusta. El primer pterigióforo de la aleta anal, el cual es notablemente largo y curvo, hace contacto con la primera espina hemal caudal y algunos otros pterigióforos anales.

El riñón mesonéfrico, el cual es extraperitonal está localizado entre las vértebras precaudales y el estómago. Las gónadas se sitúan entre el estómago y el primer pterigióforo anal; los testículos son pequeños, mientras que los ovarios son largos y se desarrollan extravisceralmente hacia atrás entre la musculatura caudal y los pterigióforos anales.

En el resto de la cavidad visceral se encuentran el tubo digestivo, el hígado, el páncreas y el bazo. Mientras que el hígado se extiende principalmente por el lado izquierdo del tubo digestivo, el bazo, el cual es pequeño se observa por el lado derecho, cerca de los ciegos pilóricos.

\section{Tubo Digestivo}

El esófago en ambas especies de Syacium en estudio es relativamente corto, de sección ovalada y hace contacto con el riñón, el seno venoso del corazón y el hígado (Fig. 1c). La interpretación del patrón de enrollamiento del tubo digestivo se muestra en la Figura $8 b$.

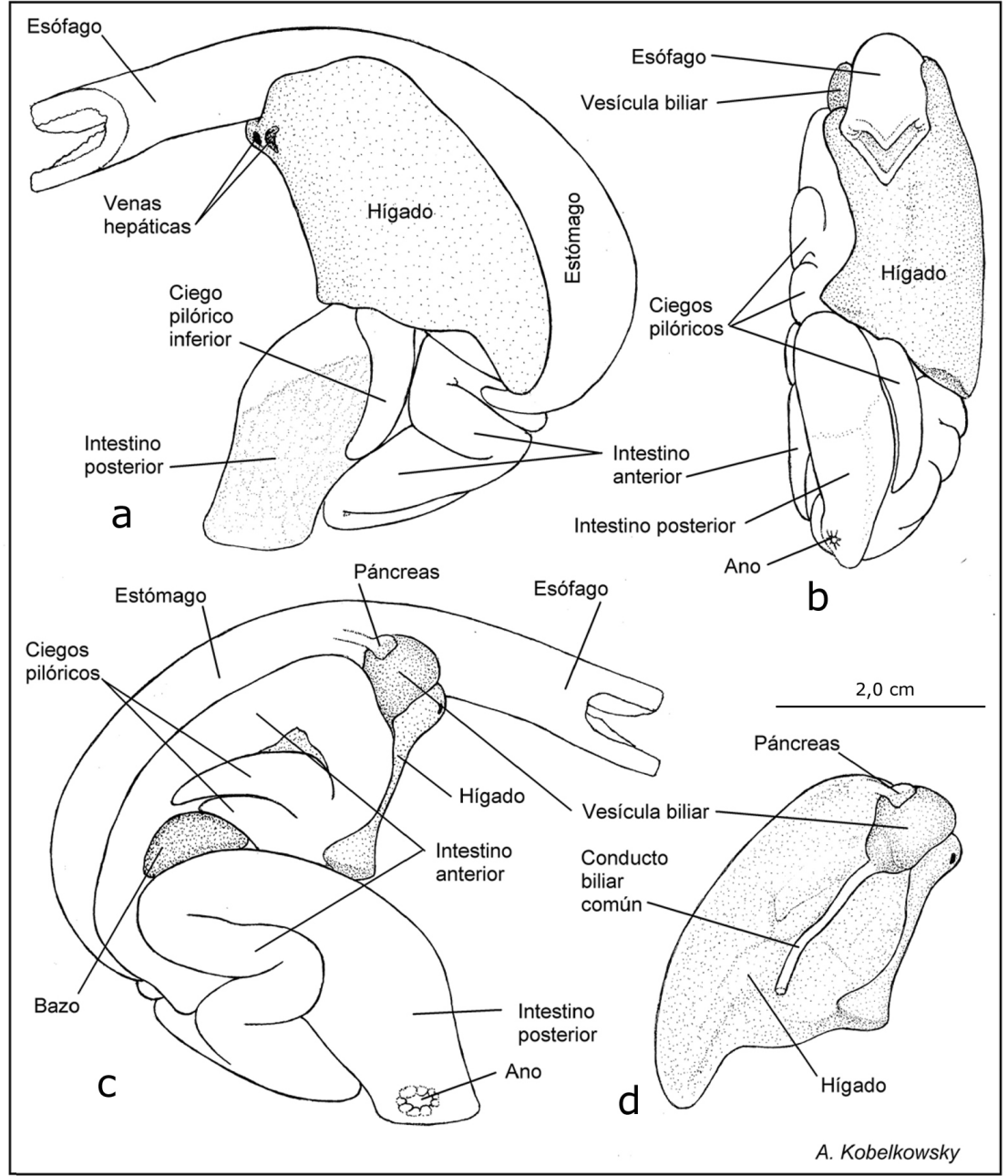

Figura 7. Tubo digestivo y glándulas anexas de Syacium papillosum. a) Vista del lado oculado, b) Vista anterior, c) Vista del lado ciego, d) Vista del lado ciego del hígado, la vesicular biliar y el páncreas / Digestive tube and anexed glands of Syacium papillosum. a) Eye side view, b) Anterior view, c) Blind side view, d) View of the blind side of the liver, gall bladder and pancreas 
El estómago es amplio, en forma de C (Figs. 1c, 8, 9a, 10c) y desarrolla ventralmente un pequeño ciego orientado hacia adelante; está parcialmente cubierto por el hígado en su lado izquierdo, hace un amplio contacto por su superficie dorsal con el riñón y un contacto menor por su parte posterior con las gónadas.

Al nivel del duodeno se desarrollan 4 ciegos pilóricos (Figs. $7,8,10 \mathrm{~d}, \mathrm{c}$ ), de los cuales 3 son cortos, orientados hacia atrás y dispuestos por el lado derecho del estómago y otro es relativamente largo y orientado hacia abajo por el lado izquierdo. El segundo ciego pilórico hace contacto con el bazo, el cual es ovoide y se observa por el lado derecho de la masa intestinal (Fig. 7c).
El intestino se divide en anterior y posterior mediante la denominada válvula intestinal (Fig. 8b). El intestino anterior es relativamente largo y de diámetro menor al del estómago; forma una amplia asa en forma de $\mathrm{C}$, paralela al costado derecho del estómago (Fig. 7c, 10e), después de la cual se forman otras asas pequeñas. El intestino posterior es grueso, relativamente corto, de posición vertical (Figs. 8b, 10d, e) y termina en un reducido recto. El ano se abre en el lado ciego, cerca del inicio de la aleta anal, inmediatamente anterior a la papila urinaria de la hembra (Fig. 1b) y a la papila urogenital del macho.

Se determinó la relación intestinal de $S$. papillosum en 1,06 y en $S$. gunteri de 0,97 .

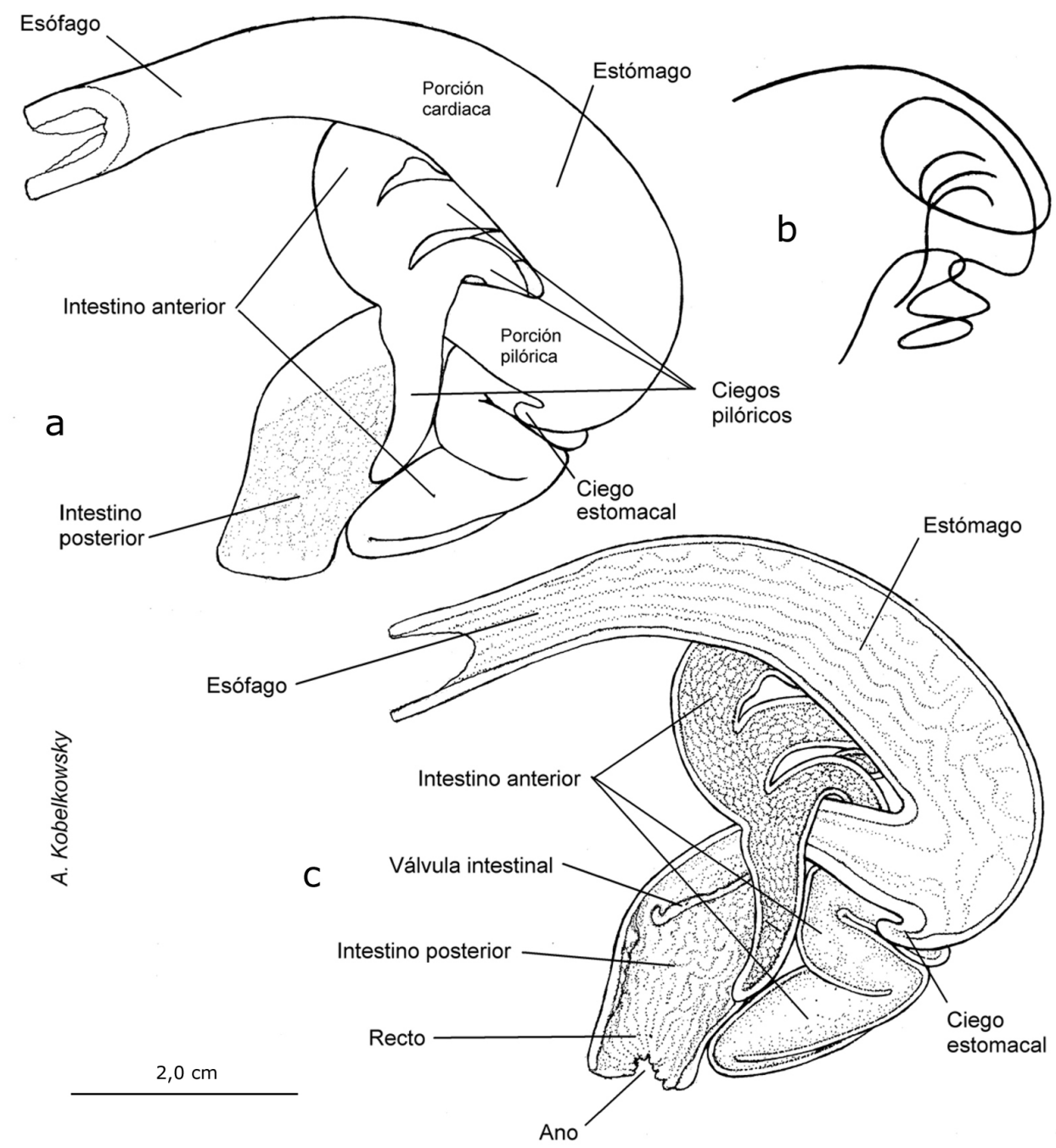

Figura 8. Tubo digestivo de Syacium papillosum. a) Vista del lado oculado, b) Patrón de enrollamiento, c) Corte longitudinal en vista del lado oculado / Digestive tube of Syacium papillosum. a) Blind side view, b) Coil pattern, c) Longitudinal section in blind side view 
El hígado es relativamente plano, se extiende por el costado izquierdo del estómago (Figs. 7a, 10b) y por la parte anterior a los 3 ciegos pilóricos derechos. Las venas hepáticas (Fig. 7a) se comunican directamente con el seno venoso del corazón. La vesícula biliar se localiza en la parte anterosuperior derecha del estómago.

El páncreas es relativamente pequeño y se localiza adosado a la superficie posterior de la vesícula biliar (Fig. 7c, d), con su conducto orientado hacia atrás, siguiendo en parte la trayectoria del estómago.

La superficie interna del tubo digestivo muestra rasgos diferentes en cada una de sus porciones (Fig. 8b). La superficie interna del esófago está formada por pliegues y surcos finos longitudinales. La superficie interna de la porción cardiaca del estómago está formada por pliegues y surcos longitudinales gruesos, mientras que en la porción pilórica son difusos. La superficie interna, tanto de los ciegos pilóricos como de la primera porción del intestino está formada por finos hexágonos, mientras que el resto del intestino anterior es de aspecto liso. Marcando el límite entre intestino anterior y posterior se encuentra la válvula intestinal, de aspecto laminar y orientada hacia abajo. La superficie interna del intestino posterior está formada por pliegues finos ondulados, mientras que en la superficie del recto son longitudinales.

\section{Aspectos Comparativos DEL SISTEMA DIGESTIVo DE S. PAPILLOSUM Y S. GUNTERI}

Los aspectos anatómicos del sistema digestivo que mostraron mayores diferencias corresponden a los ciegos pilóricos y al estómago. El ciego pilórico inferior (Figs. 7a, b) de $S$. papillosum es notablemente más alargado que el correspondiente de $S$. gunteri (Figs. 10d, e); el estómago de S. papillosum muestra un ciego estomacal (Figs. 7a, 11a), mientras que el de $S$. gunteri no lo desarrolla (Figs. 10d, 11b).

\section{Aspectos comparativos de $S$. papillosum y $S$. GUNTERI CON OTRAS ESPECIES DE LA FAMILIA Paralichthyidae}

El análisis anatómico comparativo de las especies de Syacium con Citharichthys spilopterus, Cyclopsetta chittendeni y Paralichthys lethostigma permite definir un mismo patrón morfológico del sistema digestivo de las 4 especies de Paralichthyidae. Sin embargo, de acuerdo con la Tabla 1 se identifican algunas diferencias, consistentes en el tamaño relativo de la boca y los dientes mandibulares, el número de músculos levatores operculi, la inserción del retractor dorsalis, la morfología de las branquiespinas, la presencia del ciego estomacal, el número de asas intestinales, el número de ciegos pilóricos, y la morfología de la válvula intestinal.

Tabla 1. Comparación del sistema digestivo de especies de Syacium, Citharichthys, Cyclopsetta y Paralichthys (Familia Paralichtyidae) / Comparison of the digestive system of the species of Syacium, Citharichthys, Cyclopsetta, and Paralichthys (Familia Paralichtyidae)

\begin{tabular}{|c|c|c|c|c|}
\hline & $\begin{array}{l}\text { Syacium papillosum } \\
\text { Syacium gunteri }\end{array}$ & $\begin{array}{l}\text { Citharichthys } \\
\text { spilopterus }\end{array}$ & $\begin{array}{l}\text { Cyclopsetta } \\
\text { chittendeni }\end{array}$ & $\begin{array}{c}\text { Paralichthys } \\
\text { lethostigma }\end{array}$ \\
\hline Boca & $\begin{array}{l}\text { No sobrepasa el nivel } \\
\text { medio del ojo inferior }\end{array}$ & $\begin{array}{l}\text { Alcanza el límite } \\
\text { posterior del ojo } \\
\text { inferior }\end{array}$ & $\begin{array}{l}\text { sobrepasa el borde } \\
\text { posterior del ojo } \\
\text { inferior }\end{array}$ & $\begin{array}{l}\text { Sobrepasa el borde } \\
\text { posterior del ojo } \\
\text { inferior }\end{array}$ \\
\hline Dentición mandibular & Dientes pequeños & Dientes pequeños & $\begin{array}{l}\text { Dientes del dentario } \\
\text { más lagos }\end{array}$ & $\begin{array}{l}\text { Dientes del lado ciego } \\
\text { ligeramente más largos }\end{array}$ \\
\hline Músculo levator operculi & Con una sola sección & Con una sola sección & Con una sola sección & $\begin{array}{l}\text { Dividido en dos } \\
\text { secciones }\end{array}$ \\
\hline Músculo retractor dorsalis & $\begin{array}{l}\text { Inserción en las } \\
\text { vértebras } 3 \text { a } 6\end{array}$ & $\begin{array}{l}\text { Inserción en las } \\
\text { vértebras } 2 \text { a } 4\end{array}$ & $\begin{array}{l}\text { Inserción en las } \\
\text { vértebras } 3 \text { a } 7\end{array}$ & $\begin{array}{l}\text { Inserción en las } \\
\text { vértebras } 2 \text { a } 4\end{array}$ \\
\hline Branquiespinas & $\begin{array}{l}\text { Triangulares y con una } \\
\text { hilera de dientes } \\
\text { medianos }\end{array}$ & $\begin{array}{l}\text { Delgadas y sin } \\
\text { dientes }\end{array}$ & $\begin{array}{l}\text { Ovaladas y con una } \\
\text { hilera de dientes } \\
\text { grandes }\end{array}$ & $\begin{array}{l}\text { Triangulares y con una } \\
\text { hilera de dientes } \\
\text { medianos }\end{array}$ \\
\hline Estómago & $\begin{array}{l}\text { En forma de } \mathrm{C} \text {, con } \\
\text { ciego estomacal }\end{array}$ & $\begin{array}{l}\text { En forma de } C \text {, sin } \\
\text { ciego estomacal }\end{array}$ & $\begin{array}{l}\text { En forma de } C \text {, sin } \\
\text { ciego estomacal }\end{array}$ & $\begin{array}{l}\text { En forma de } \mathrm{C}, \sin \\
\text { ciego estomacal }\end{array}$ \\
\hline Intestino & Con 6 asas & Con 3 asas & Con 3 asas & \\
\hline Ciegos pilóricos & En número de 4 & En número de 4 & En número de 3 & En número de 4 \\
\hline Válvula intestinal & En forma de tabique & Con forma circular & Con forma circular & Con forma circular \\
\hline
\end{tabular}




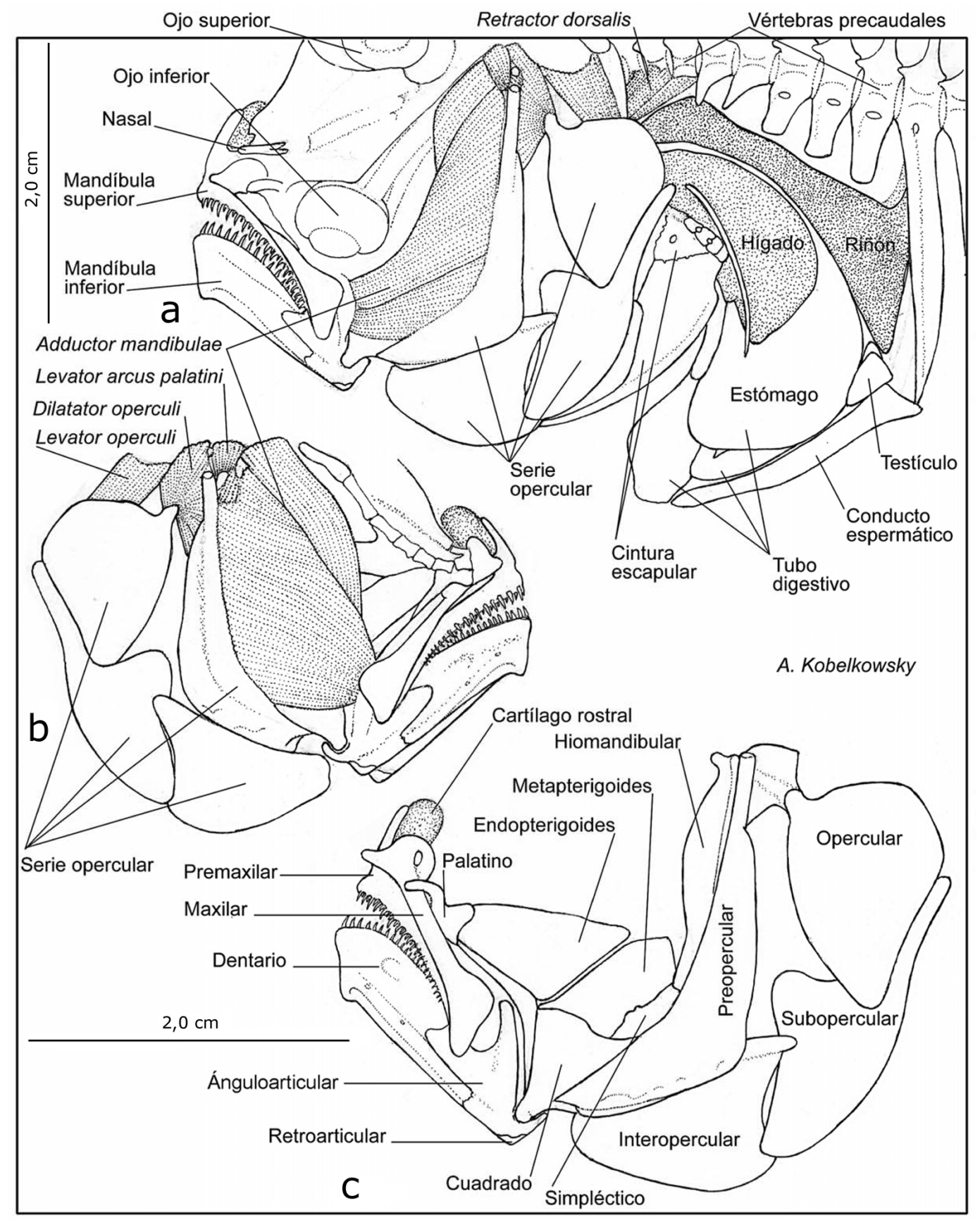

Figura 9. Sistema digestivo de Syacium gunteri. a) Vista del lado oculado de parte del esqueleto visceral, la musculatura visceral y de la cavidad visceral, b) Vista del lado ciego de la región cefálica, c) Vista del lado oculado de las series mandibular, palatina, opercular y suspensión mandibular / Digestive system of Syacium gunteri. a) Eye side view of part of the visceral skeleton and musculature, b) Blind side view of the cephalic region, c) Eye side view of the mandibular, palatine and opercular series, and the jaw suspension 

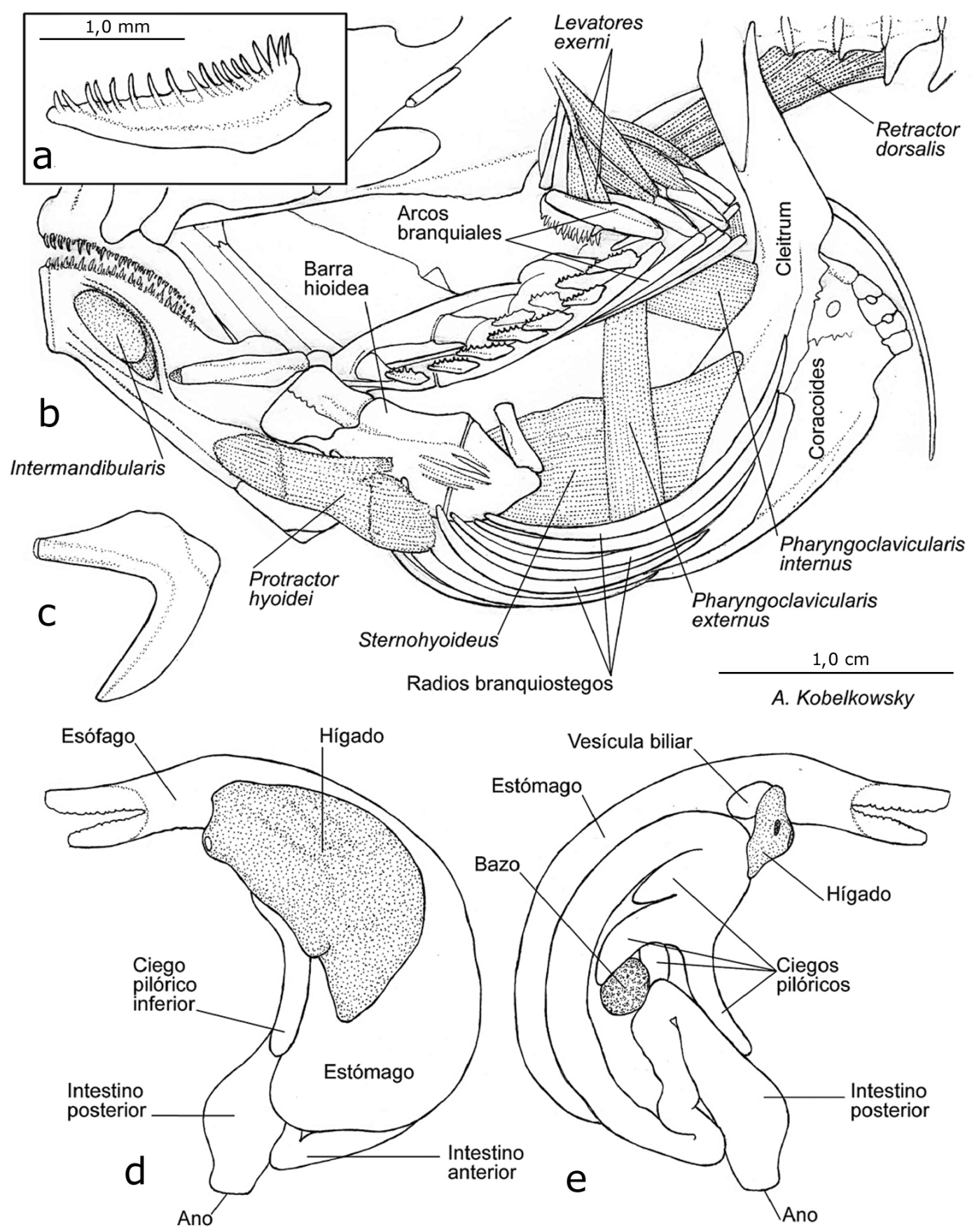

Figura 10. Esqueleto branquial, musculatura branquial y tubo digestivo de Syacium gunteri. a) Branquiespina del primer arco branquial, b) Vista del lado oculado del esqueleto branquial y hioideo, y parte de su musculatura, c) Vista lateral izquierda del urohial, d) Vista del lado oculado del tubo digestivo, e) Vista del lado ciego del tubo digestivo / Branchial skeleton and musculature, and digestive tube of Syacium gunteri. a) Gill raker of the first branchial arch, b) Blind side view of the branchial and hyoid skeleton, and of part of their musculature, c) Left lateral view of urohyal, d) Eye side view of the digestive tube, e) Blind side view of the digestive tube 

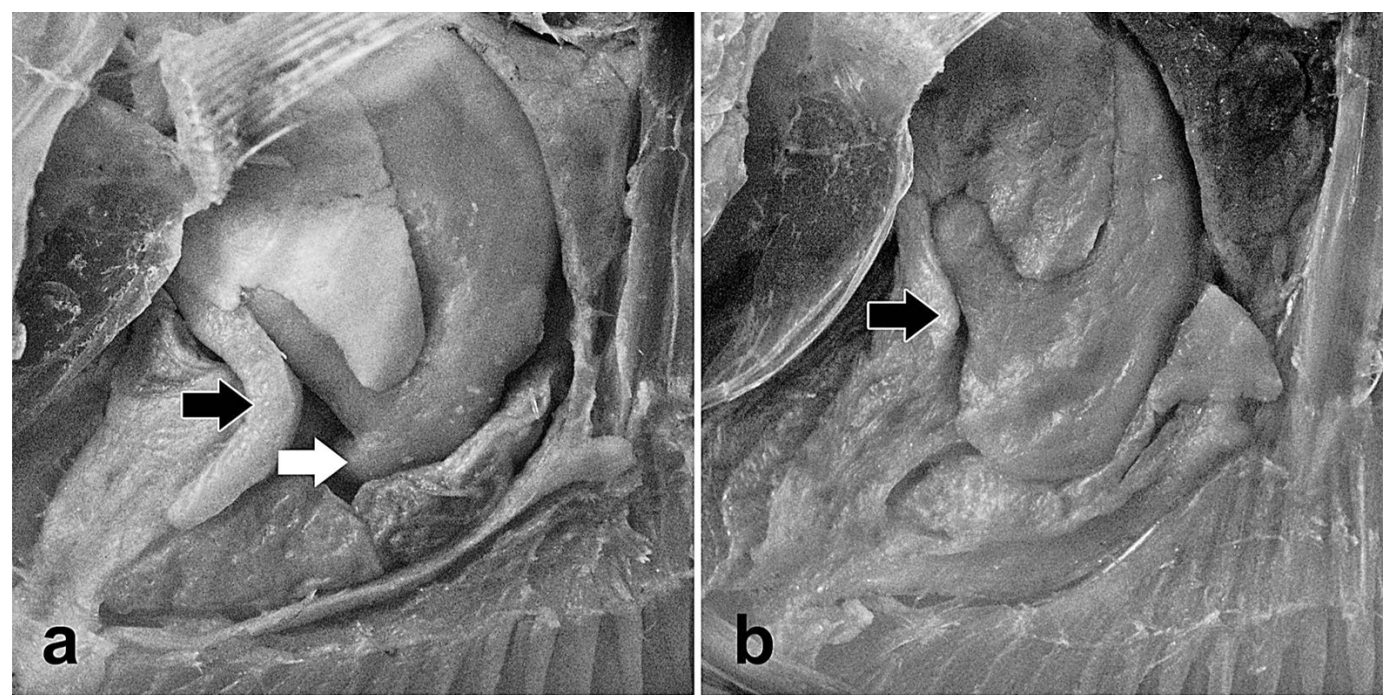

Figura 11. Principales diferencias anatómicas del tubo digestivo entre Syacium papillosum y Syacium gunteri. Vista del lado oculado. a) S. papillosum mostrando el ciego pilórico inferior (flecha negra) y el ciego estomacal (flecha blanca), b) S. gunteri mostrando el ciego pilórico inferior (flecha negra) / Main anatomical differences of the digestive tube between Syacium papillosum and Syacium gunteri. Eye side. a) $\mathrm{S}$. papillosum showing the lower pyloric cecum (black arrow) and the stomach cecum (white arrow), b) S. gunteri showing the lower pyloric cecum (black arrow)

\section{Aspectos comparativos de las especies de Paralichthyidae con representantes de las familias Bothidae, Achiridae y Cynoglossidae (Tabla 2, Fig. 12)}

En el representante de la familia Bothidae, Bothus robinsi la boca es corta (Fig. 12a), la longitud de las mandíbulas del lado ciego es ligeramente mayor que la del lado oculado, el arreglo de la dentición mandibular es simétrico (Fig. 12b), el músculo adductor mandibulae es más amplio en el lado ciego, las branquiespinas son cortas y sin dientes, el estómago tiene forma de $\mathrm{C}$ y no desarrolla ciego estomacal (Fig. 12c); el intestino anterior forma varias asas, después de la última se conforma el intestino posterior, el cual es amplio.

En el representante de la familia Achiridae, Achirus lineatus la boca es notablemente asimétrica (Fig. 12d), siendo más curvada en el lado ciego y la dentición mandibular solo se encuentra en el premaxilar y el dentario del lado ciego (Fig. 12e); no se forman verdaderas branquiespinas; el estómago tiene forma de $\mathrm{C}$ y no desarrolla ciego estomacal; el carácter más sobresaliente se observa en la ubicación de 2 asas intestinales extraviscerales que se extienden por el lado oculado del pez, acompañando al ovario derecho (Fig. 12f); no se desarrollan ciegos pilóricos y no se define el intestino posterior.

En el representante de la familia Cynoglossidae, Symphurus civitatium la boca es notablemente asimétrica (Fig. 12g), siendo más curvada en el lado ciego y la dentición mandibular (Fig. 12h) se encuentra en el premaxilar y el dentario del lado ciego, mientras que en el lado oculado solo se observan escasos dientes en el premaxilar; el rasgo más importante es la suspensión del tubo digestivo al nivel del esófago (Fig. 12i) mediante una fascia que lo une con los procesos hemales de las vértebras precaudales 4 a 6; no se desarrollan ciegos estomacal ni ciegos pilóricos y no se define el intestino posterior.

\section{DiscusIón}

Mientras que la mayoría de las publicaciones sobre la anatomía del sistema digestivo de los teleósteos, restringe su descripción a la forma de la boca y del tubo digestivo, el presente estudio incluye tanto la del esqueleto visceral como la de musculatura visceral de Syacium papillosum y Syacium gunteri. De acuerdo con Wilson \& Castro (2011), la cavidad bucofaríngea tiene la función de obtener el alimento y procesarlo mecánicamente, mientras que la del esófago y el estómago es iniciar la digestión química del alimento y la del intestino es continuar la digestión y realizar la absorción del mismo.

A diferencia de numerosos teleósteos la morfología del sistema digestivo de ambas especies de Syacium y probablemente también de los restantes Pleuronectiformes, está determinada principalmente por 3 factores: a) el acentuado aplanamiento lateral del cuerpo, b) la asimetría de la región cefálica y c) la reducción del volumen de la cavidad visceral. 
Tabla 2. Comparación del sistema digestivo de especies representativas de las familias Paralichthyidae (Syacium papillosum, Syacium gunteri, Citharichthys spilopterus, Cyclopsetta chittendeni, Paralichthys lethostigma), Bothidae (Bothus robinsi), Achiridae (Achirus lineatus) y Cynoglossidae (Symphurus civitatium) / Comparison of the digestive system of representative of the families Paralichthyidae (Syacium papillosum, Syacium gunteri, Citharichthys spilopterus, Cyclopsetta chittendeni, Paralichthys lethostigma), Bothidae (Bothus robinsi), Achiridae (Achirus lineatus), and Cynoglossidae (Symphurus civitatium)

\begin{tabular}{|c|c|c|c|c|}
\hline & Paralichthyidae & Bothidae & Achiridae & Cynoglossidae \\
\hline Forma de la boca & $\begin{array}{l}\text { Ligeramente curvada } \\
\text { en ambos lados }\end{array}$ & $\begin{array}{l}\text { Ligeramente curvada } \\
\text { en ambos lados }\end{array}$ & $\begin{array}{l}\text { Más curvada en el } \\
\text { lado ciego }\end{array}$ & $\begin{array}{l}\text { Más curvada en el } \\
\text { lado ciego }\end{array}$ \\
\hline Dentición mandibular & $\begin{array}{l}\text { Similar en premaxilar } \\
\text { y dentario de ambos } \\
\text { lados }\end{array}$ & $\begin{array}{l}\text { Similar en premaxilar } \\
\text { y dentario de ambos } \\
\text { lados }\end{array}$ & $\begin{array}{l}\text { Presente en } \\
\text { premaxilar y dentario } \\
\text { del lado ciego. } \\
\text { Ausente en las } \\
\text { mandíbulas del lado } \\
\text { oculado }\end{array}$ & $\begin{array}{l}\text { Presente en ambos } \\
\text { premaxilares y en el } \\
\text { dentario del lado } \\
\text { ciego. Ausente en el } \\
\text { dentario del lado } \\
\text { oculado }\end{array}$ \\
\hline $\begin{array}{l}\text { Longitud relativa de las } \\
\text { mandíbulas }\end{array}$ & $\begin{array}{l}\text { Mandíbulas superior } \\
\text { e inferior del lado } \\
\text { ciego, ligeramente } \\
\text { más largas }\end{array}$ & $\begin{array}{l}\text { Mandíbulas superior } \\
\text { e inferior del lado } \\
\text { ciego, ligeramente } \\
\text { más largas }\end{array}$ & $\begin{array}{l}\text { Mandíbulas superior } \\
\text { e inferior del lado } \\
\text { ciego, más grandes }\end{array}$ & $\begin{array}{l}\text { Mandíbulas superior } \\
\text { e inferior del lado } \\
\text { ciego, más grandes }\end{array}$ \\
\hline $\begin{array}{l}\text { Simetría del adductor } \\
\text { mandibulae }\end{array}$ & $\begin{array}{l}\text { Más amplio en el } \\
\text { lado ciego }\end{array}$ & $\begin{array}{l}\text { Más amplio en el } \\
\text { lado ciego }\end{array}$ & $\begin{array}{l}\text { Ligeramente más } \\
\text { amplio en el lado } \\
\text { ciego }\end{array}$ & $\begin{array}{l}\text { Ligeramente más } \\
\text { amplio en el lado } \\
\text { ciego }\end{array}$ \\
\hline Branquiespinas & Presentes & Presentes & Ausentes & Ausentes \\
\hline Estómago & $\begin{array}{l}\text { Grande y en forma de } \\
\text { C }\end{array}$ & $\begin{array}{l}\text { Grande y en forma de } \\
\text { C }\end{array}$ & $\begin{array}{l}\text { Grande y en forma de } \\
\text { C }\end{array}$ & Largo y recto \\
\hline Intestino & $\begin{array}{l}\text { Corto y sin asas } \\
\text { extraviscerales }\end{array}$ & $\begin{array}{l}\text { Corto y sin asas } \\
\text { extraviscerales }\end{array}$ & $\begin{array}{l}\text { Largo y con dos asas } \\
\text { extraviscerales en el } \\
\text { lado oculado }\end{array}$ & $\begin{array}{l}\text { Largo y sin asas } \\
\text { extraviscerales }\end{array}$ \\
\hline Ciegos pilóricos & 3 o 4 & 3 o 4 & Ausentes & Ausentes \\
\hline Válvula intestinal & Presente & Presente & Ausente & Ausente \\
\hline $\begin{array}{l}\text { Origen del retractor } \\
\text { dorsalis }\end{array}$ & En las vértebras 3 a 6 & En las vértebras 3 a 5 & En las vértebras 2 a 4 & En las vértebras 2 a 4 \\
\hline
\end{tabular}

El pronunciado aplanamiento lateral del cuerpo de los lenguados implica el aplanamiento en el mismo sentido de las cavidades bucofaríngea y visceral. Las series de huesos que conforman las mandíbulas, el paladar, la suspensión de las mandíbulas, el opérculo, el aparato hioideo y en menor grado, el aparato branquial, están dispuestas principalmente en el plano vertical. Asimismo, numerosos músculos que mueven a estas series son aplanados lateralmente.

La asimetría del esqueleto visceral se reconoce principalmente en las series palatinas, siendo los palatinos y los endopterigoides los huesos más asimétricos. Tal situación morfológica ha sido descrita e ilustrada por Díaz de Astarloa (2005) en 3 especies de Paralichthys (Paralichthyidae) y Flores \& De la Hoz (2010) en Hippoglossina macrops (Paralichthyidae). La asimetría de los endopterigoides se manifiesta por la concavidad del hueso del lado oculado que contribuye a formar una nueva órbita ocular, descrita en Citharichthys spilopterus por Kobelkowsky (2002) y denominada en Cyclopsetta chittendeni como 'órbita ocular secundaria' por Kobelkowsky (2007). La asimetría se observa en las mandíbulas, las suspensiones mandibulares y las series operculares, pero no se percibe en el aparato hioideo. En el aparato branquial la asimetría consiste en la posición alternada de los huesos faringobranquiales 2 a 4, con los dientes faríngeos superiores orientados hacia atrás, lo cual supone una deglución efectiva. Los músculos viscerales que muestran mayor asimetría son el adductor mandibulae, con un mayor volumen el del lado ciego, y el intermandibularis con la base del lado ciego más amplia. La mayor amplitud del adductor mandibulae del lado ciego se correlaciona con la ausencia en ese lado del ojo migratorio. 

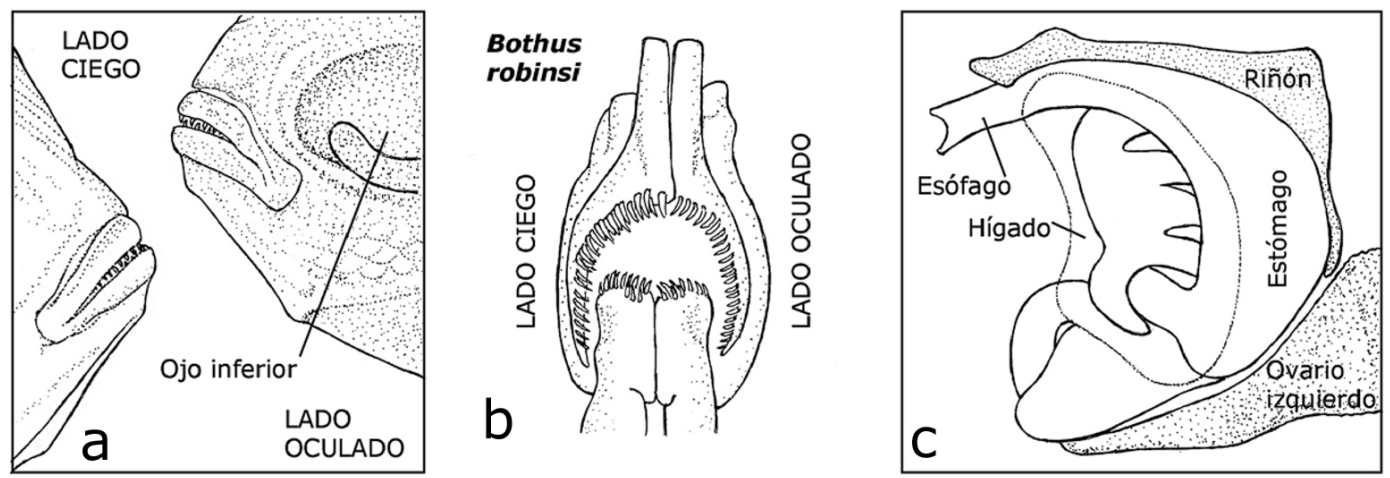

A. Kobelkowsky
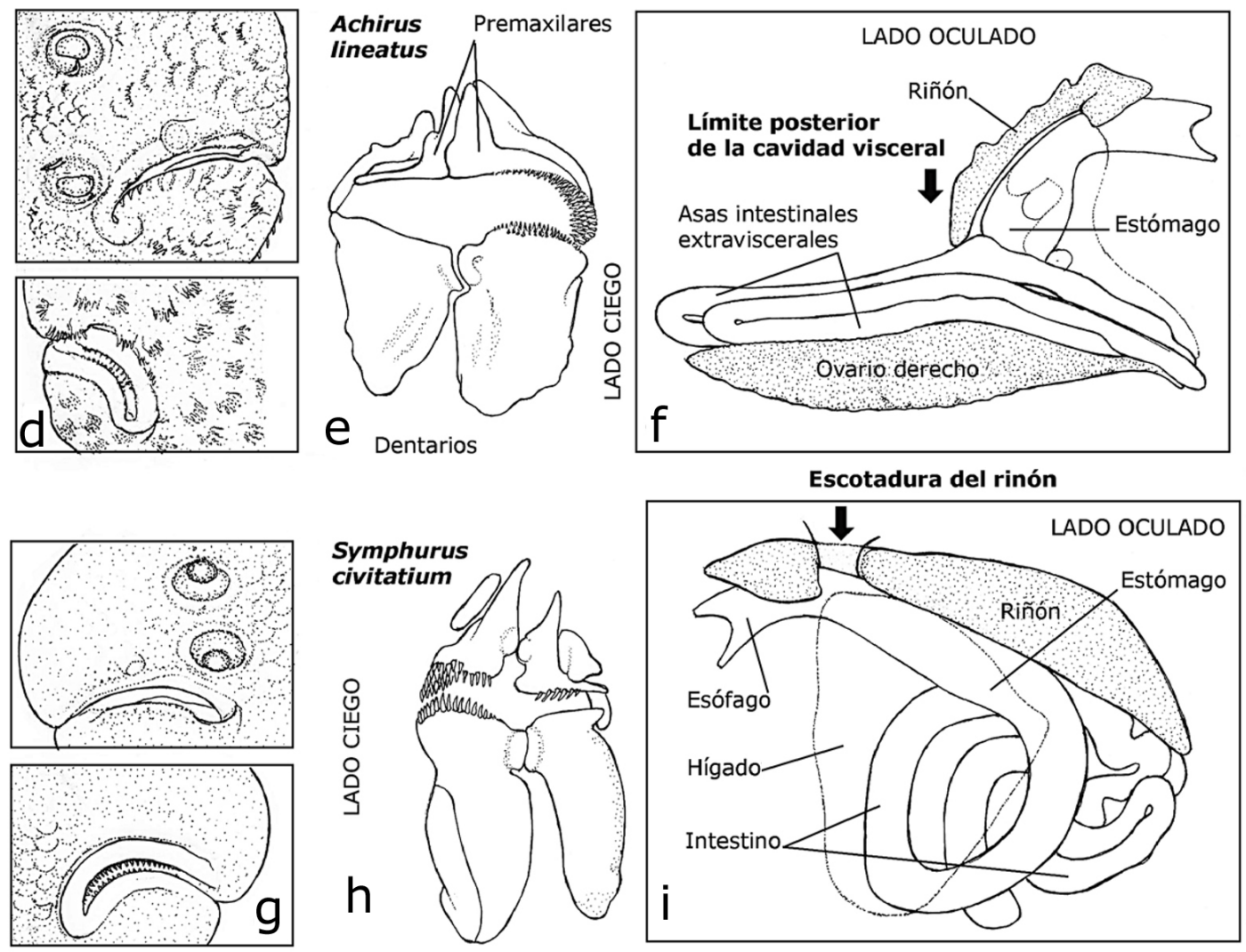

Figura 12. Caracteres anatómicos sobresalientes del sistema digestivo de representantes de las familias Bothidae, Achiridae y Cynoglossidae. a) Vista de la boca de los lados ciego y oculado de Bothus robinsi, b) Vista anterior de las mandíbulas de B. robinsi, c) Vista del lado oculado del tubo digestivo de B. robinsi, d) Vista de la boca de los lados oculado y ciego de Achirus lineatus, e) Vista anterior de las mandíbulas de A. lineatus, $\mathrm{f}$ ) Vista del lado oculado del tubo digestivo de A. lineatus, g) Vista de la boca de los lados oculado y ciego de Symphurus civitatium, h) Vista anterior de las mandíbulas de S. civitatium, i) Vista del lado oculado del tubo digestivo de S. civitatium / Outstanding anatomical characters of the digestive system of representative species of the families Bothidae, Achiridae, and Cynoglossidae. a) View of the mouth of both blind and eye sides of Bothus robinsi, b) Anterior view of the jaws of B. robinsi, c) Eye side view of the digestive tube of B. robinsi, d) View of the mouth of both sides of Achirus lineatus, e) Anterior view of the jaws of $A$. lineatus, $f$ ) Eye side view of the digestive tube of $A$. lineatus, g) View of the mouth of both sides of Symphurus civitatium. h) Anterior view of the jaws of S. civitatium, i) Eye side view of the digestive tube of S. civitatium 
La asimetría de las mandíbulas consistente en la mayor longitud de las del lado ciego y la mayor robustez del adductor mandibulae de dicho lado, probablemente implique una apertura asimétrica de la boca, como es descrita por Gibb (2003) en Pleuronichthys verticalis (Pleuronectidae). Van Dobben (1935) describe en los Pleuronectiformes la orientación asimétrica de la boca durante la captura de la presa, siendo más notable en los Soleidae y Cynoglossidae, en los cuales indica que el lado oculado de la boca permite la circulación del agua para el intercambio gaseoso, mientras que el lado ciego se utiliza para la captura de la presa.

La reducción del tamaño de la cavidad visceral es debida al aplanamiento lateral del cuerpo y al adelantamiento de la aleta anal. Probablemente dicha reducción ha sido un proceso gradual, que ya se percibía en el lenguado primitivo Heteronectes chaneti del Eoceno (Friedman 2012). El adelantamiento de la aleta anal de S. papillosum y $S$. gunteri, así como también de los restantes Pleuronectiformes implica el crecimiento extravisceral de los ovarios y la ubicación del ano y las aberturas urogenitales en una posición notablemente adelantada, como lo describen Kobelkowsky (2000a) en Achirus lineatus (Achiridae), Kobelkowsky (2000b) en Symphurus civitatium (Cynoglosidae), Kobelkowsky (2004) en Bothus robinsi (Bothidae) y Kobelkowsky (2007) en C. chittendeni (Paralichthyidae), y como se aprecia en el presente estudio en Paralichthys lethostigma (Paralichthyidae). El aspecto más notable de la organografía de la cavidad visceral de los lenguados es la ubicación extravisceral de 2 asas intestinales en los Achiridae; en segundo término, la suspensión del esófago desde algunas vértebras en los Cynoglossidae.

La organización del tubo digestivo de ambas especies de Syacium corresponde en términos generales a la de la mayoría de los teleósteos, reconociéndose las regiones del esófago, estómago, ciegos pilóricos, intestino anterior e intestino posterior. Sin embargo, el hábito carnívoro de alimentación se correlaciona con el mayor tamaño del estómago, como en la mayoría de los Pleuronectiformes, con excepción de los Rhombosoleidae Ammotretis rostrata y Rhombosolea tapirina descritas por Grove \& Campbell (1979) como especies sin estómago ni ciegos pilóricos.

Mientras que en varios teleósteos carnívoros de tipo depredador el estómago forma un largo ciego estomacal, que alberga la presa mientras se realiza la digestión, en $S$. papillosum resalta la formación de un pequeño ciego estomacal cerca de la región pilórica, mientras que en $S$. gunteri no se desarrolla esta estructura.

Así como en otros teleósteos, las especies en estudio muestran la formación de la válvula intestinal que divide al intestino en anterior y posterior. De acuerdo con Mok (1980) los Pleuronectiformes tienen un tipo de enrollamiento intestinal consistente en un asa intestinal dirigida hacia delante y otra hacia atrás, sin embargo, en S. papillosum y $S$. gunteri así como también en C. spilopterus (Castillo-Rivera et al. 2000), $C$. chittendeni (Kobelkowsky 2007), B. robinsi y P. lethostigma, el último tramo intestinal se orienta hacia delante y se dobla formando el intestino posterior.

La organización anatómica del sistema digestivo de $S$. papillosum y $S$. gunteri corresponde a especies de teleósteos carnívoros de tipo bentófago, lo cual coincide con lo señalado para todos los Pleuronectiformes por autores como Norman (1934) y Podoskina (1993).

La organización anatómica del esqueleto visceral, la musculatura visceral y la dentición de $S$. papillosum y $S$. gunteri se encuentra dentro de la principal estrategia trófica de los acantopterigios o teleósteos superiores, que de acuerdo con Vanderwalle et al. (2000) y Wainright \& Bellwood (2002) consiste en la captura de la presa, que generalmente es por succión y su procesamiento en la cavidad faríngea.

Aunque no existen registros de la dieta de S. papillosum, probablemente su alimentación sea similar a la de $S$. gunteri (García-Abad et al. 1992), consistente en crustáceos peneidos y pequeños peces y en menor grado de poliquetos.

Las largas mandíbulas y la forma aguda de sus dientes permiten la ingestión de presas relativamente grandes. La dentición de las branquiespinas, probablemente produzca una descamación de las presas ícticas en teleósteos depredadores (Moyle \& Cech Jr. 2000). De Groot (1971) indica que los lenguados ingieren completa su presa y la digieren casi totalmente en el estómago, el cual es grande. La presencia de los ciegos pilóricos probablemente contribuya a la absorción del alimento (Buddington \& Diamond 1987). Los escasos estudios del sistema digestivo de lenguados, registran una cierta diversidad del número de ciegos pilóricos; así en su estudio filogenético de los Scophthalmidae, Chanet (2003) señala su ausencia en Phrynorhombus regius y Zeugopterus punctatus, mientras que en Psetta maxima registra el desarrollo de 2 ciegos pilóricos; Castillo-Rivera et al. (2000) registran 4 ciegos pilóricos en C. spilopterus, y Kobelkowsky (2007) cuenta 3 ciegos en C. chittendeni.

El análisis anatómico comparativo del sistema digestivo de las especies de Syacium, C. spilopterus, C. chittendeni y $P$. lethostigma contribuye a la definición de un patrón morfológico del sistema digestivo de la familia Paralichthyidae. Asimismo, el análisis comparativo de los Paralichthyidae con especies representativas de las familias Bothidae, Achiridae y Cynoglossidae permite reconocer preliminarmente una mayor afinidad morfológica del sistema digestivo con los Bothidae, que con los Achiridae y los Cynoglossidae. 


\section{LITERATURA CITADA}

Buddington RK \& JM Diamond. 1987. Pyloric ceca of fish: a 'new' absorptive organ. American Journal of Physiology 252: 65-76.

Castillo-Rivera M, A Kobelkowsky \& AM Chávez. 2000. Feeding biology of the flatfish Citharichthys spilopterus (Bothidae) in a tropical estuary of Mexico. Journal of Applied Ichthyology 16: 73-78.

Chanet B. 2003. Interrelationships of Scophthalmid fishes (Pleuronectiformes: Scophthalmidae). Cybium 27(4): 275-286.

Chittenden ME \& JD McEachran. 1976. Composition, ecology and dynamics of demersal fish communities in the north-western Gulf of Mexico continental shelf, with a similar synopsis for the entire Gulf. Sea Grant Publications TAMUSG-76-208: 1-104.

De Groot SJ. 1971. On the interrelationships between morphology of the alimentary tract, food and feeding behaviour in flatfishes (Pisces: Pleuronectiformes). Netherlands Journal of Sea Research 5: 121-196.

Díaz de Astarloa JM. 2005. Osteología craneal comparada de tres especies de lenguado del género Paralichthys (Pleuronectiformes, Paralichthyidae) del Atlántico suroccidental. Revista Chilena de Historia Natural 78: 343 391.

Figueiredo JL \& NA Menezes. 2000. Manual de Peixes Marinhos do Sudeste do Brasil. VI. Teleostei 5, 116 pp. Museu de Zoologia. Universidade de Sao Paulo, Sao Paulo.

Flores H \& E De la Hoz. 2010. Osteología de Hippoglossina macrops (Pleuronectiformes, Paralichthyidae). Revista de Biología Marina y Oceanografía 45(1): 547-563.

Friedman M. 2012. Osteology of Heteronectes chaneti (Acanthomorpha, Pleuronectiformes), an Eocene stem flatfish, with a discussion of flatfish sister-group relationships. Journal of Vertebrate Paleontology 32: 735-756.

García-Abad MC, A Yáñez-Arancibia, P Sánchez-Gil \& M Tapia García. 1992. Distribución, reproducción y alimentación de Syacium gunteri Ginsburg (Pisces: Bothidae), en el Golfo de México. Revista de Biología Tropical 39(1): 27-34.

Gibb AC. 2003. Modeling the jaw mechanism of Pleuronichthys verticalis: the morphological basis asymmetrical jaws movement in a flatfish. Journal of Morphology 256: 1-12.

Grove DJ \& G Campbell. 1979. The role of extrinsic nerves in the coordination of gut motility in the stomachless flatfish Rhombosolea tapirina and Ammotretis rostrata Guenther. Comparative Biochemistry \& Physiology 63C: 143-159.

Hoshino K \& K Amaoka. 1998. Osteology of the flounder, Tephrinectes sinensis (Lacépède) (Teleostei: Pleuronectiformes), with comments on its relationships. Ichthyological Research 45: 69-77.

Kobelkowsky A. 2000a. Sistema urogenital de los lenguados de la familia Achiridae (Pisces: Pleuronectiformes) del Golfo de México. Hidrobiológica 10: 51-60.
Kobelkowsky A. 2000b. Sistema digestivo del lenguado Symphurus (Pisces: Cynoglossidae). Zoología Informa ENCB-IPN 43: 17-26.

Kobelkowsky A. 2002. Osteología del lenguado Citharichthys spilopterus (Pisces: Pleuronectiformes). Anales del Instituto de Biología, Universidad Nacional Autónoma de México, Serie Zoología 73: 53-65.

Kobelkowsky A. 2004. Sexual dimorphism of the flounder Bothus robinsi (Pisces: Bothidae). Journal of Morphology 260: 165-171.

Kobelkowsky A. 2007. Morphology of the digestive system of the Mexican flounder, Cyclopsetta chittendeni (Teleostei, Paralichthyidae). Bulletin of Fish Biology 9: 39-49.

Mok H-K. 1980. Notes on the classification of actinopterigian intestinal patterns. Japanese Journal of Ichthyology 27(1): 29-40.

Moyle PB \& JJ Cech Jr. 2000. Fishes: An introduction to ichthyology, 590 pp. Prentice Hall, Upper Saddle River.

Murakami T \& K Amaoka. 1992. Review of the genus Syacium (Paralichthyidae) with the description of a new species from Ecuador and Colombia. Bulletin of the Faculty of Fisheries Hokkaido University 43(2): 61-95.

Nelson JS. 2006. Fishes of the world, 601 pp. John Wiley \& Sons, Hoboken.

Nikolsky GV. 1963. The ecology of fishes, 352 pp. Academic Press, London \& New York.

Norman JR. 1934. A systematic monograph of the flatfishes (Heterosomata). Vol. 1, Psettodidae, Bothidae, Pleuronectidae, 480 pp. British Museum of Natural History, London.

Podoskina TA. 1993. Morphology of some pleuronectiform fishes in relation to feeding specializations. Journal of Ichthyology 246: 116-122.

Policansky D. 1982. The asymmetry of flounders. Scientific American 246: 116-122.

Prasada Rao PD \& TE Finger. 1984. Asymmetry of the olfactory system in the brain of the winter flounder, Pseupleuronectes americanus. The Journal of Comparative Neurology 225: 492-510.

Topp RW \& FH Hoff Jr. 1972. Flatfishes (Pleuronectiformes). Memoirs of the Hourglass Cruises, Florida Marine Research Institute Department of Natural Resources 4(1): 1-135.

Van Dobben WH. 1935. Uber den Kiefermechanismus der Knochenfische. Archives Néerlandaises de Zoologie 2: 172.

Wilson JM \& LFC Castro. 2011. Morphological diversity of the gastrointestinal tract in fishes. In: Grosell M, AP Farrell \& CJ Brauer (eds). The multifunctional gut of fish, pp. 2-55. Academic Press, London.

Winterbottom R. 1974. A descriptive synonymy of striated muscles of the Teleostei. Proceeding of the Academy of Natural Sciences of Philadelphia 125: 225-317. 